\title{
A Survey of Commercially Available Manipulators, End-Effectors, and Delivery Systems for Reactor Decommissioning Activities
}

\section{Decontamination and Decommissioning Program}

Argonne National Laboratory 9700 South Cass Avenue

Argonne, Illinois 60439
Operated by The University of Chicago, under Contract W-31-109-ENG-38, for the United States Department of Energy 


\section{Argonne National Laboratory}

Argonne National Laboratory, with facilities in the states of Illinois and Idaho, is owned by the United States Government, and operated by the University of Chicago under the provisions of a contract with the Department of Energy.

This technical report is a product of Argonne's Technology Development Division, Decontamination and Decommissioning Program. For information on the division's activities, contact:

Director, Technology Development Division

Argonne National Laboratory

Argonne, Illinois 60439-4815

Telephone (708) 252-6050

Publishing support services were provided by Argonne's Information and Publishing Division (for more information, see IPD's home page: http://www.ipd.anl.gov/).

\section{Disclaimer}

This report was prepared as an account of work sponsored by an agency of the United States Government. Neither the United States Government nor any agency thereof, nor any of their employees, makes any warranty, express or implied, or assumes any legal liability or responsibility for the accuracy, completeness, or usefulness of any information, apparatus, product, or process disclosed, or represents that its use would not infringe privately owned rights. Reference herein to any specific commercial product, process, or service by trade name, trademark, manufacturer, or otherwise, does not necessarily constitute or imply its endorsement, recommendation, or favoring by the United States Government or any agency thereot. The views and opinions of authors expressed herein do not necessarily state or reflect those of the United States Government or any agency thereof. available to DOE and DOE contractors from the Office of Scientific and Technical Information, P.O. Box 62, Oak Ridge, TN 37831; prices avallable from (423) $576-8401$.

Available to the public from the National Technical Information Service, U.S. Department of Commerce, 5285 Port Royal Road, Springfield, VA 22161. 
DISCLAIMER

Portions of this document may be illegible in electronic image products. Images are produced from the best available original document. 


\section{A Survey of Commercially Available Manipulators, End-Effectors, and Delivery Systems for Reactor Decommissioning Activities}

by Donald R. Henley and Thomas J. Litka*

Decontamination and Decommissioning Program, Technology Development Division, Argonne National Laboratory, 9700 South Cass Avenue, Argonne, Illinois 60439

May 1996

Work sponsored by United States Department of Energy,

Office of Environmental Management

*Litka is affiliated with Advanced Consulting Group, Incorporated, Chicago, Illinois. 
This report is printed on recycled paper. 


\section{CONTENTS}

SUMMARY 1

1 INTRODUCTION 3

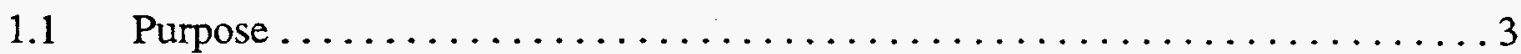

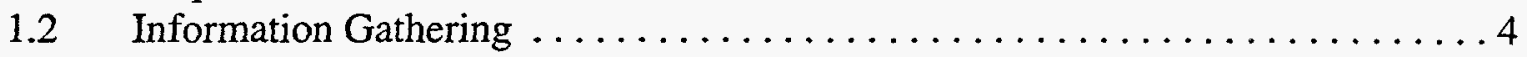

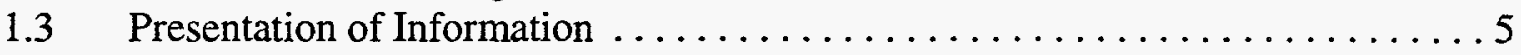

2 COMMERCIAL AVAILABILITY OF EQUIPMENT $\ldots \ldots \ldots \ldots \ldots \ldots \ldots$

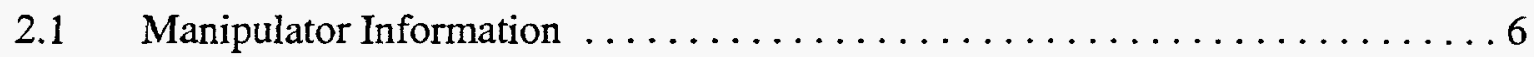

2.1.1 Suppliers Contacted and Categories $\ldots \ldots \ldots \ldots \ldots \ldots \ldots \ldots$

2.1.2 Specifications and Cost Estimates $\ldots \ldots \ldots \ldots \ldots \ldots \ldots \ldots$

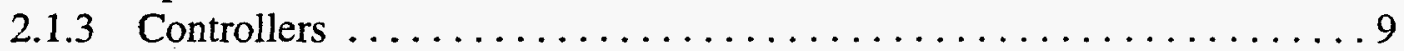

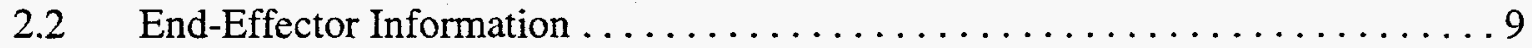

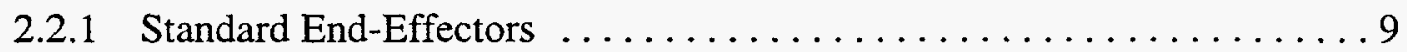

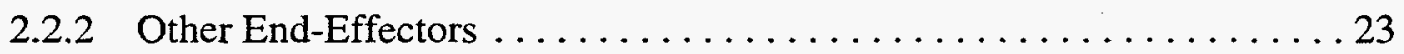

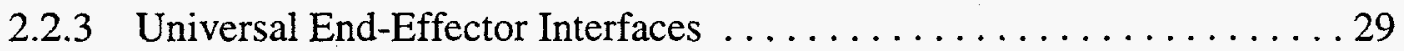

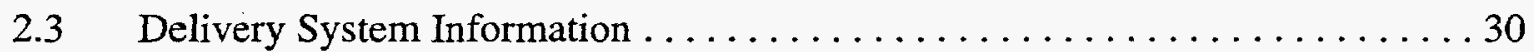

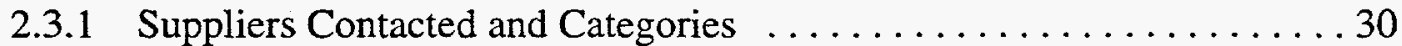

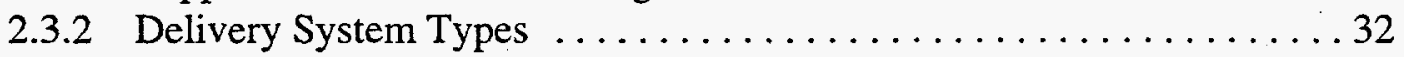

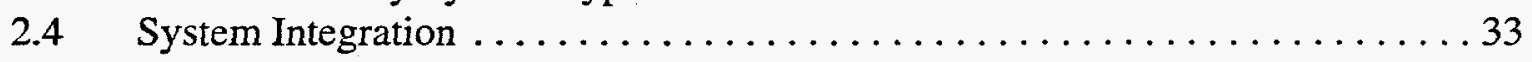

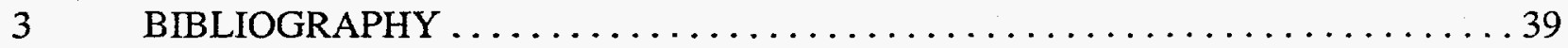

APPENDIX A: MANIPULATOR SUPPLIERS $\ldots \ldots \ldots \ldots \ldots \ldots \ldots \ldots \ldots \ldots \ldots \ldots \ldots \ldots$

APPENDIX B: CRANE AND GANTRY SUPPLIERS $\ldots \ldots \ldots \ldots \ldots \ldots \ldots \ldots \ldots$

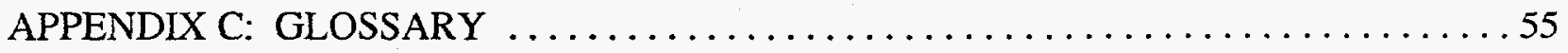

\section{TABLES}

1 Categories and Numbers of Suppliers of Manipulators $\ldots \ldots \ldots \ldots \ldots \ldots \ldots$

2 Category RA1 - Specifications of Hydraulically Powered Manipulators $\ldots \ldots \ldots 10$

3 Category RA2 - Specifications of Hydraulically Powered Manipulators $\ldots \ldots \ldots 17$

4 Category RA1 - Specifications of Electrically Powered Manipulators ........... 19 


\section{TABLES (Cont.)}

5 Category RA2 - Specifications of an Electrically Powered Manipulator $\ldots \ldots \ldots 25$

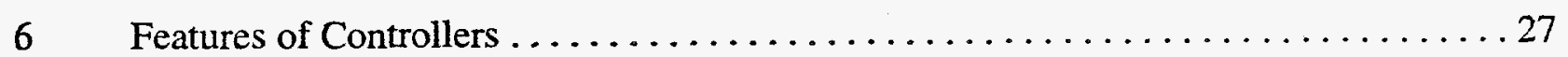

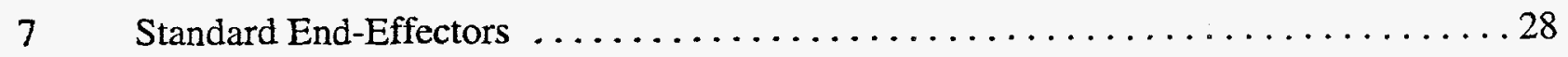

$8 \quad$ Categorization and Description of Suppliers of Crane/Gantry Equipment .......... 31

9 Category CG1 Crane/Gantry Equipment — Design and Build: Full Range ........ 35

10 Category CG2 Crane/Gantry Equipment — Design and Build: Up to 50 Tons ..... 35

11 Category CG3 Crane/Gantry Equipment — Hydraulic Jack Gantries ........... 36

12 Category CG4 Crane/Gantry Equipment — Standard Product: Special Use . . . . . . 36

13 Category CG5 Crane/Gantry Equipment - Booms and Lifts $\ldots \ldots \ldots \ldots \ldots \ldots$

A.1 Categories Used to Describe Robotic Arms $\ldots \ldots \ldots \ldots \ldots \ldots \ldots \ldots \ldots$

B.1 Categories Used to Describe Cranes/Gantries ....................... 51

\section{FIGURES}

1 Typical Arrangement of a Manipulator, End-Effector, and Bridge Crane $\ldots \ldots \ldots \ldots 4$

2 BM3000 Manipulator, Manufactured by Brokk, North American Sales, Inc. . . . . . 13

3 Conan T Manipulator, Manufactured by GEC Alsthom Schilling Robotic Systems, Inc. ................................ 14

$4 \quad$ Hydra T Manipulator, Manufactured by GEC Alsthom Schilling Robotic Systems, Inc. ............................... 14

$5 \quad$ RigMaster Manipulator, Manufactured by GEC Alsthom Schilling Robotic Systems, Inc.

6 Titan III T Manipulator, Manufactured by GEC Alsthom Schilling Robotic Systems, Inc.

7 RMS 2500 Series Manipulator, Manufactured by Spar Aerospace Limited 18 


\section{FIGURES (Cont.)}

$8 \quad$ MLDUA Manipulator, Manufactured by Spar Aerospace Limited $\ldots \ldots \ldots \ldots \ldots 18$

9 Model 3350 Manipulator, Manufactured by PaR Systems, Inc. . . . . . . . . 21

10 Model 4350 Manipulator, Manufactured by PaR Systems, Inc. . . . . . . . . 22

11 Model 6350 Manipulator, Manufactured by PaR Systems, Inc. ............ 23

12. Helios Manipulator - Manufactured by GEC Alsthom Schilling

Robotic Systems, Inc. ................................. 24

13 TeleMate Manipulator, Manufactured by Omniview, Inc. $\ldots \ldots \ldots \ldots \ldots \ldots 26$

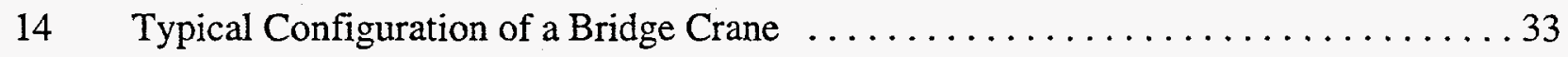

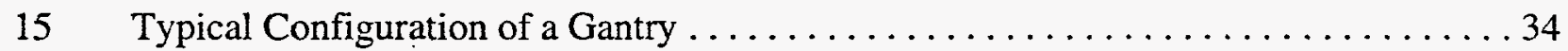

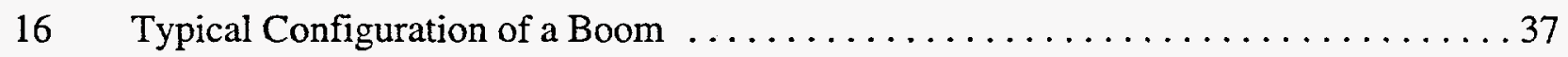

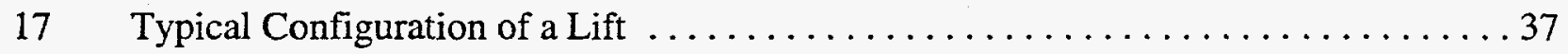




\title{
A SURVEY OF COMMERCIALLY AVAILABLE \\ MANIPULATORS, END-EFFECTORS, AND \\ DELIVERY SYSTEMS FOR REACTOR \\ DECOMMISSIONING ACTIVITIES
}

\author{
by \\ D.R. Henley and T.J. Litka
}

\begin{abstract}
SUMMARY
Numerous nuclear facilities owned by the U.S. Department of Energy (DOE) are under consideration for decommissioning. Currently, there are no standardized, remote, automated systems designed to dismantle and thereby reduce the size of activated reactor components and vessels so that they can be packaged and shipped to disposal sites. Existing dismantling systems consist of customized tooling that has been developed to dismantle a specific reactor system. A standardized, remote, automated system is therefore needed to deliver the specific tooling required for dismantling reactor systems at different locations.
\end{abstract}

A standardized dismantling system is expected to be the most cost-effective means of dismantling and removing reactors. The system requires one or more manipulators (i.e., robotic arms), multiple end-effectors, and a delivery system. The manipulators with end-effectors can perform various tasks, such as cutting and removing reactor components. Numerous manipulators are available. Some standard end-effectors are available, but many are unique. The delivery system for the manipulator most likely would be a gantry or overhead crane, but other types of booms and lifts should be considered. Delivery systems (such as cranes and gantries) are usually custom designed and built for a specific application.

A standardized dismantling system would provide the DOE community, commercial utilities, and the public with many benefits. For example, the system could be designed for the decommissioning and decontamination of fuel storage pools, hot cells, and fuel-reprocessing canyons. To save money and improve reliability, the new system should be based on currently available hardware, which can be procured from private industry. The tasks of fabricating and supplying dismantling systems will ultimately be transferred to the private sector.

The purpose of this document is to survey the commercially available equipment that could be assembled to produce such a universal dismantling system. To provide this information, Argonne National Laboratory contacted more than 70 suppliers of manipulators and cranes/gantries. Argonne 
categorized the suppliers and presents them in this report. In addition, Argonne provides some detailed information about each manipulator, as well as a list of standard end-effectors. 


\section{INTRODUCTION}

\subsection{PURPOSE}

Numerous nuclear facilities owned by the U.S. Department of Energy (DOE) are under consideration for decommissioning. Currently, there are no standardized, automated, remote systems designed to dismantle and thereby reduce the size of activated reactor components and vessels so that they can be packaged and shipped to disposal sites. Existing dismantling systems usually consist of customized, facility-specific tooling that has been developed to dismantle a specific reactor system. Such systems have a number of drawbacks. Generally, current systems cannot be disassembled, moved, and reused. Developing and deploying the tooling for current systems is expensive and timeconsuming. In addition, the amount of manual work is significant because long-handled tools must be used; as a result, personnel are exposed to excessive radiation.

A standardized, automated, remote system is therefore needed to deliver the tooling necessary to dismantle nuclear facilities at different locations. Because this system would be reusable, it would produce less waste. The system would also save money because of its universal design, and it would be more reliable than current systems.

An industry objective is to develop a universal system for dismantling reactors and removing reactor components. Such a system would be the most cost-effective approach to decommissioning nuclear facilities. To be effective, each component of the dismantling system should be commercially available (or as close to being commercially available as possible) and exemplify the latest technology. The dismantling system would probably consist of a manipulator, multiple end-effectors, and a delivery system (see Figure 1). The manipulator and end-effectors would perform various tasks, such as cutting and removing reactor components. The delivery system would most likely consist of a mast, an overhead crane, or a gantry, but other types of booms and lifts or motorized vehicles might also be appropriate. Each of these delivery systems has unique advantages and disadvantages.

Such a standardized dismantling system could provide the DOE community, commercial utilities, and the public with many benefits. For example, the system could be designed for the decommissioning and decontamination of fuel storage pools, hot cells, and fuel-reprocessing canyons. To save money and improve reliability, a new dismantling system should be based on commercially available hardware. The tasks of fabricating, supplying, and deploying dismantling systems will ultimately be transferred to the private sector. 


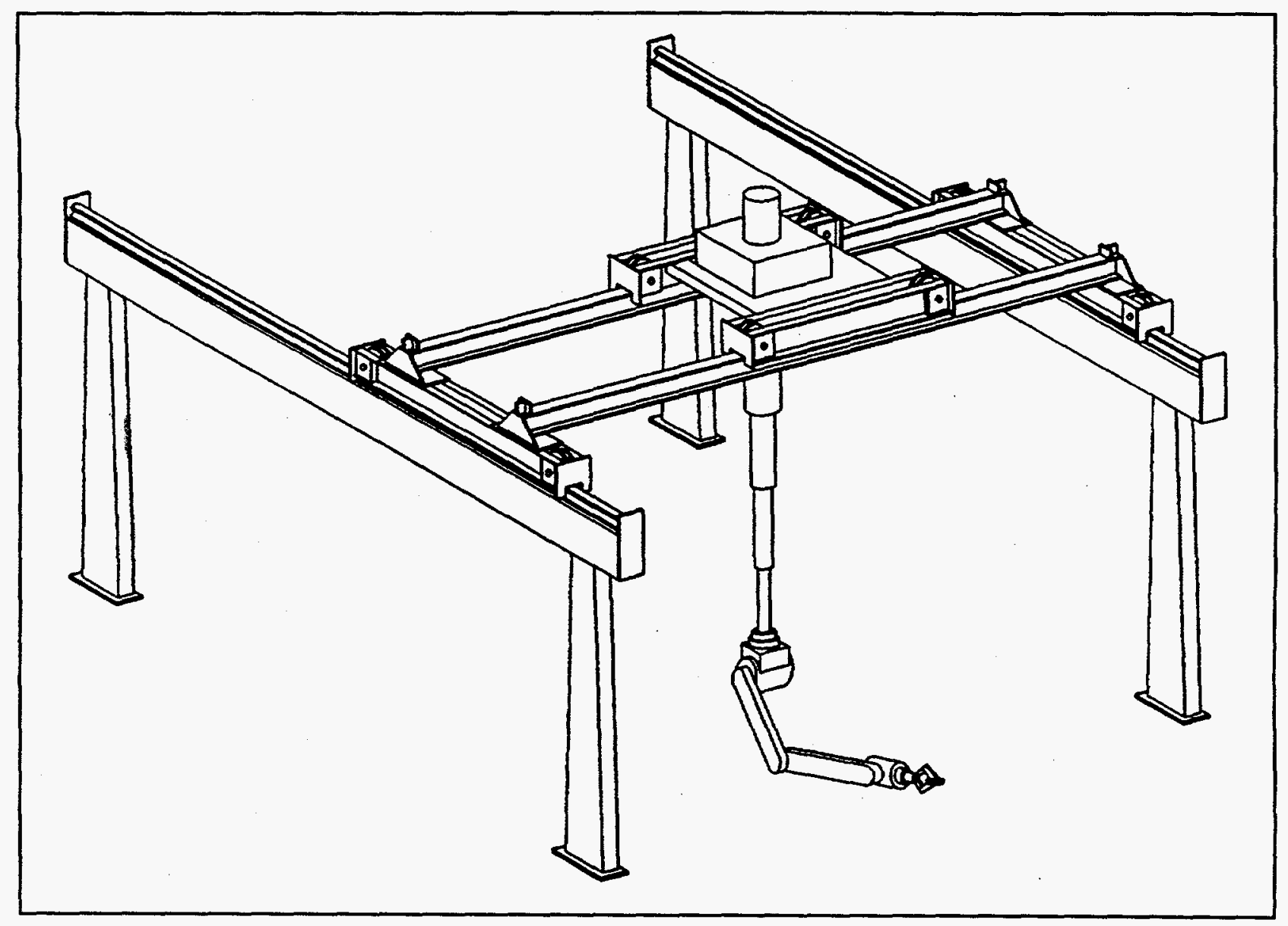

FIGURE 1 Typical Arrangement of a Manipulator, End-Effector, and Bridge Crane

\subsection{INFORMATION GATHERING}

Argonne National Laboratory (ANL), through funding by the Office of Technology Development (EM-50), has surveyed the manufacturers of commercial equipment that might be used to perform remote decommissioning and decontamination tasks at DOE facilities. More than 70 suppliers were contacted for information. Every effort was made to make this survey as comprehensive as possible. To help achieve that goal, the suppliers were all asked to review the information presented in the tables in this report.

To gather information for this report, Argonne:

- Gathered and reported information on all applicable equipment,

- Used commercial nuclear industry purchasing reference publications to identify suppliers (see publications 1 through 4 in the Bibliography), 
- Investigated non-nuclear suppliers on the basis of referrals from the nuclear suppliers, and

- Contacted U.S. suppliers and foreign suppliers with U.S.-based offices.

An additional source of information on robotics is the Internet. Some particularly useful resources are listed below.

- Robotics Internet Resources Page: http://piglet.cs.umass.edu:4321/robotics.html.

- NASA's Jet Propulsion Laboratory Robotics Homepage: http://robotics.jpl.nasa.gov.

- Robotics Frequently Asked Questions List: http://www.frc.cmu.edu:80/robotics-faq.

This last homepage provides a link to a very extensive list of companies that manufacture and sell robotic equipment.

\subsection{PRESENTATION OF INFORMATION}

All of the data obtained are presented in Section 2 of this report. The report consists of sections that describe manipulators, end-effectors, and delivery systems. Information about suppliers of manipulators and delivery systems is divided into subsections. Controls are discussed in the section on manipulators. Information is summarized in tables whenever possible so that equipment can be compared easily. Cost estimates are given only if they were provided by the suppliers and are included for information only. 


\section{COMMERCIAL AVAILABILITY OF EQUIPMENT}

\subsection{MANIPULATOR INFORMATION}

\subsubsection{Suppliers Contacted and Categories}

Numerous types of manipulators are available for reactor decommissioning. Nuclear industry purchasing publications list many suppliers as being involved with remote handling/robotics. Some of these suppliers have commercially available manipulators for procurement, but many do not. Robotic industrial welding arms are also available, but they are not described in this report.

Argonne contacted the 54 suppliers listed in Appendix A to determine if they had commercially available manipulators. After reviewing the information provided by the suppliers, Argonne developed a categorization that describes the industry. The categories and numbers of suppliers of manipulators are listed in Table 1. Note that several suppliers provide equipment in more than one category.

TABLE 1 Categories and Numbers of Suppliers of Manipulators

\begin{tabular}{llc}
\hline Category & \multicolumn{1}{c}{ Description } & $\begin{array}{c}\text { Number of } \\
\text { Suppliers }\end{array}$ \\
\hline RA1 & Commercially available manipulators with controls & 8 \\
RA2 & $\begin{array}{l}\text { Provide manipulators that have been used several times but have } \\
\text { not been offered as commercial (near-commercial status) }\end{array}$ & 2 \\
RA3 & Develop unique manipulators for an application & 4 \\
RA4 & Purchase manipulators and package them for an application & 10 \\
RA5 & Provide crawlers and/or remotely operated vehicles (ROVs) & 14 \\
RA6 & Develop and provide controls for manipulators only & 2 \\
RA7 & Do not offer any robotic equipment or services & 19 \\
\hline
\end{tabular}


The suppliers of manipulators in categories RA1 through RA6 are listed below (for more information on each supplier, see Section 4):

\title{
RA1 Commercially Available Manipulators with Controls
}

AEA Technology, Pittsburgh, PA (United Kingdom)

BNFL, Inc., Walnut Creek, CA (United Kingdom)

Brokk, Seattle, WA (Sweden)

GEC Alsthom Schilling Robotic Systems, Inc., Davis, CA (United Kingdom)

Kraft TeleRobotics, Inc., Overland Park, KS

PaR Systems, Inc., Shoreview, MN

Sarcos, Inc., Salt Lake City, UT

Western Space and Marine, Santa Barbara, CA

RA2 Provided a Manipulator Several Times

("near commercial")

Omniview, Inc., Knoxville, TN

Spar Aerospace Limited, Avon, CT (Canada)

\section{RA3 Develop Unique Manipulators for Specific Applications}

\author{
AECL Technologies, Rockville, MD (Canada) \\ ASI Robotics Systems, Clawgon, MI \\ Nuclear Systems Associates, Brea, CA \\ Odetics, Anaheim, CA
}

RA4 Purchase Manipulators and Package Them for Specific Applications

The Bartholomew Co., Inc., Westbury, NY

General Atomics, San Diego, CA

NAC International, Norcross, GA

NES, Inc., Danbury, CT

NUS, Clearwater, FL

Oceaneering Technologies, Rockville, MD

PLS International, Cleveland, $\mathrm{OH}$ 
RedZone Robotics, Inc., Inc., Pittsburgh, PA

Resource Services International, Cleveland, $\mathrm{OH}$

Rust Utility Services, Inc., Essex, CT

\section{RA5 Provide Crawlers or ROVs}

AECL Technologies, Rockville, MD (Canada)

ARD Corp., Columbia, MD

Benthos, Inc., N. Falmouth, MA

Brokk, Seattle, WA (Sweden)

Cybermotion, Inc., Salem, VA

Foster-Miller, Inc., Waltham, MA

Grove Worldwide, Shady Grove, PA

Kraft TeleRobotics, Inc., Overland Park, KS

Remotec, Oak Ridge, TN

ROV Technologies, Inc., Vernon, CT

Spar Aerospace Limited, Avon, CT (Canada)

Viking Systems International, Pittsburgh, PA

Visual Inspection Technologies, Flanders, NJ

Western Space and Marine, Santa Barbara, CA

\section{RA6 Provide Controls for Manipulators Only}

Deneb Robotics, Inc., Auburn Hills, MI

Silma, Inc., Cupertino, CA

In addition to companies that produce conventional manipulators, Central Research Laboratories (Redwing, MN) and R.W. Wiesener, Inc. (Albermarle, NC) provide hot-cell, throughwall manipulators. These types of manipulators have not been addressed in this study.

Most suppliers of manipulators in Categories RA1 through RA4 are also system integrators that use commercial manipulators, gantries, cranes, or other positioners all operated from a single controller. These suppliers purchase equipment if available, design it if it is not available, and supply the controls needed for the application. They may also develop special end-effectors. 


\subsubsection{Specifications and Cost Estimates}

Detailed information can be provided only for manipulators in categories RA1 and RA2.

\subsubsection{Hydraulically Powered Manipulators}

The suppliers of Categories RA1 and RA2 equipment have provided specifications and cost estimates for hydraulically powered manipulators. This information is given in Tables 2 and 3.

\subsubsection{Electrically Powered Manipulators}

The suppliers of Categories RA1 and RA2 equipment have provided the specifications and cost estimates for electrically powered manipulators. This information is given in Tables 4 and 5 .

\subsubsection{Controllers}

A controller is required to control the motions and path of each robotic element. The simplest controller is a manually operated hydraulic or electrical joystick. More sophisticated controllers are usually computer-based and have such features as programmed cutting paths, teachand-learn capabilities, force feedback, and collision avoidance. Computer-based controllers can usually control more than one manipulator, and they can coordinate the motion of a manipulator, a crane, a gantry, or a mast. Because the various controllers vary in sophistication, their prices often vary by more than a factor of 10 . Table 6 lists the features of the controllers supplied by several suppliers.

\subsection{END-EFFECTOR INFORMATION}

\subsubsection{Standard End-Effectors}

A number of standard end-effectors are commercially available. Argonne asked roboticequipment suppliers to provide information about their end-effectors. Some suppliers have what they consider to be "standard" end-effectors. All equipment suppliers indicated that end-effectors are often unique and are developed and packaged for specific applications. Survey information will be presented only for end-effectors considered to be standard products. Only 6 of the 13 Category RA1, RA2, and RA3 suppliers of manipulators have standard end-effectors. Even these standard 
TABLE 2 Category RA1 - Specifications of Hydraulically Powered Manipulators (Commercially Available)

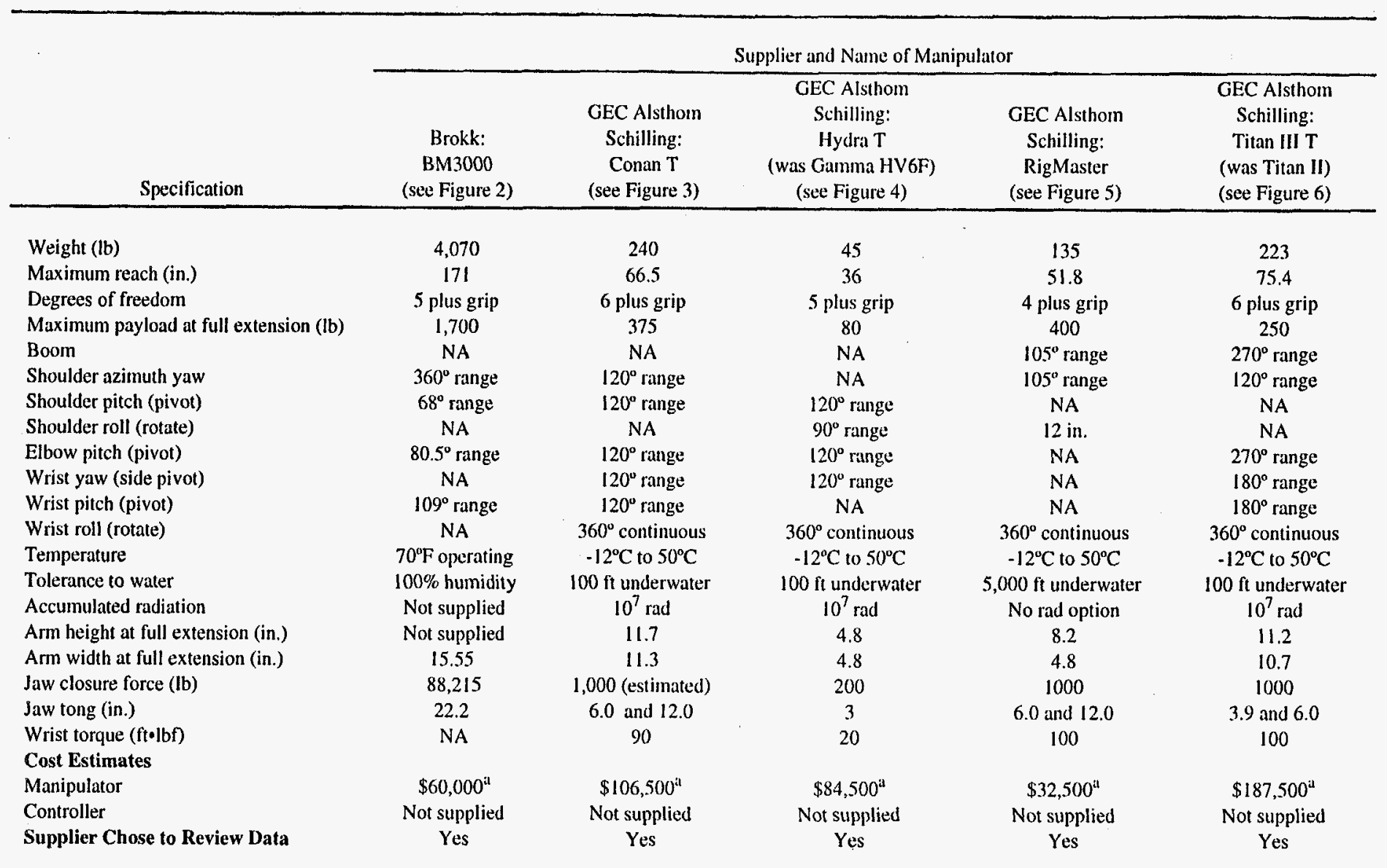


TABLE 2 (Cont.)

Supplier and Name of Manipulator

\begin{tabular}{|c|c|c|c|c|c|}
\hline Specification & $\begin{array}{l}\text { Kraft TeleRobotics: } \\
\text { Grips } \\
\text { Force Feedback } \\
\text { Manipulator } \\
\text { (figure not supplied) }\end{array}$ & $\begin{array}{l}\text { Kraft TeleRobotics: } \\
\text { Grips II } \\
\text { Force Feedback } \\
\text { Manipulator } \\
\text { (figure not supplied) }\end{array}$ & $\begin{array}{l}\text { Kraft TeleRobotics: } \\
\text { Haz-Trak } \\
\text { Force Feedback } \\
\text { Manipulator } \\
\text { (figure not supplied) }\end{array}$ & $\begin{array}{l}\text { Kraft TeleRobotics: } \\
\text { Joystick Rate } \\
\text { Manipulator } \\
\text { (figure not supplied) }\end{array}$ & $\begin{array}{l}\text { Kraft TeleRobotics: } \\
\text { Nine-Function } \\
\text { Manipulator } \\
\text { (figure not supplied) }\end{array}$ \\
\hline Weight (lb) & 130 & 150 & 2,800 & 130 & 164 \\
\hline Maximum reach (in.) & 51 & 54 & 192 & 66 & 69 \\
\hline Degrees of freedom & 6 plus grip & 6 plus grip & 6 plus grip & 6 plus grip & 8 plus grip \\
\hline Maximum payload at full extension (lb) & 100 & 100 & 1,600 & 100 & 120 \\
\hline Boom & NA & NA & NA & NA & NA \\
\hline Shoulder azimuth yaw & $180^{\circ}$ range & $180^{\circ}$ range & $140^{\circ}$ range & $120^{\circ}$ range & $120^{\circ}$ range \\
\hline Shoulder pitch (pivot) & $120^{\circ}$ range & $120^{\circ}$ range & $110^{\circ}$ range & $120^{\circ}$ range & $120^{\circ}$ range \\
\hline Shoulder roll (rotate) & NA & NA & NA & $190^{\circ}$ range & $200^{\circ}$ range \\
\hline Elbow pitch (pivot) & $110^{\circ}$ range & $110^{\circ}$ range & $100^{\circ}$ range & $120^{\circ}$ range & $110^{\circ}$ range \\
\hline Wrist yaw (side pivot) & $105^{\circ}$ range & $200^{\circ}$ range & $180^{\circ}$ range & $190^{\circ}$ range & $115^{\circ}$ range \\
\hline Wrist pitch (pivot) & $100^{\circ}$ range & $200^{\circ}$ range & $135^{\circ}$ range & $190^{\circ}$ range & $115^{\circ} \times 2$ range \\
\hline Wrist roll (rotate) & $360^{\circ}$ range & $360^{\circ}$ range & $360^{\circ}$ range & $360^{\circ}$ range & $360^{\circ}$ range \\
\hline Temperature & $0^{\circ}$ to $150^{\circ} \mathrm{F}$ & $0^{\circ} \mathrm{F}$ to $150^{\circ} \mathrm{F}$ & $0^{\circ} \mathrm{F}$ to $150^{\circ} \mathrm{F}$ & $0^{\circ} \mathrm{F}$ to $150^{\circ} \mathrm{F}$ & $0^{\circ} \mathrm{F}$ to $150^{\circ} \mathrm{F}$ \\
\hline Tolerance to water & $8,000 \mathrm{ft}$ underwater & $8,000 \mathrm{ft}$ underwater & $95 \%$ humidity & $8,000 \mathrm{ft}$ underwater & $8,000 \mathrm{ft}$ underwater \\
\hline Accumulated radiation & Not supplied & Not supplied & Not supplied & Not supplied & Not supplied \\
\hline Arm height at full extension (in.) & Not supplied & Not supplied & Not supplied & Not supplied & Not supplied \\
\hline Arm weight full extension (in.) & Not supplied & Not supplied & Not supplied & Not supplied & Not supplied \\
\hline Jaw closure force (lb) & 200 & 200 & 880 & 200 & 200 \\
\hline Jaw tong (in.) & 4 & 4 & 24 & 4 & 4 \\
\hline Wrist torque (ft•lbf) & 16 & 60 & 740 & 30 & 30 \\
\hline \multicolumn{6}{|l|}{ Cost Estimates } \\
\hline Manipulator & Not supplied & Not supplied & Not supplied & Not supplied & Not supplied \\
\hline Controller & Not supplied & Not supplied & Not supplied & Not supplied & Not supplied \\
\hline Supplier Chose to Review Data & Yes & Yes & Yes & Yes & Yes \\
\hline
\end{tabular}


TABLE 2 (Cont.)

Supplier and Name of Manipulator

\begin{tabular}{|c|c|c|c|c|}
\hline Sppecification & $\begin{array}{c}\text { Sarcos: } \\
\text { Dextrous Manipulator } \\
\text { (figure not supplied) }\end{array}$ & $\begin{array}{c}\text { Sarcos: } \\
\text { GRLA Manipulator } \\
\text { (figure not supplied) }\end{array}$ & $\begin{array}{c}\text { Western Space and Marine: } \\
\text { The Manipulator } \\
\text { (figure not supplied) }\end{array}$ & $\begin{array}{c}\text { Western Space and Marine: } \\
\text { MK } 37 \\
\text { (figure not supplied) }\end{array}$ \\
\hline Weight (lb) & 53 & Not supplied & 320 & 95 \\
\hline Maximum reach (in.) & 32.65 & 72 & 65 & 37 \\
\hline Degrees of freedom & 7 plus hand & 7 plus hand & 7 & 7 \\
\hline Maximum payload at full extension (lb) & 40 & 350 & 100 & 50 \\
\hline Boom & NA & NA & Not supplied & Not supplied \\
\hline Shoulder azimuth yaw & $180^{\circ}$ range & Not supplied & $180^{\circ}$ range & $180^{\circ}$ range \\
\hline Shoulder pitch (pivot) & $180^{\circ}$ range & Not supplied & $120^{\circ}$ range & $120^{\circ}$ range \\
\hline Shoulder roll (rotate) & $180^{\circ}$ range & Not supplied & NA & NA \\
\hline Elbow pitch (pivot) & $180^{\circ}$ range & Not supplied & $180^{\circ}$ range & $180^{\circ}$ range \\
\hline Wrist yaw (side pivot) & $90^{\circ}$ range & Not supplied & $115^{\circ}$ range & $120^{\circ}$ range \\
\hline Wrist pitch (pivot) & $180^{\circ}$ range & Not supplied & $100^{\circ}$ range & $100^{\circ}$ range \\
\hline Wrist roll (rotate) & $180^{\circ}$ range & Not supplied & $200^{\circ}$ range & $220^{\circ}$ range \\
\hline Temperature & Not supplied & Not supplied & $-20^{\circ} \mathrm{F}$ to $230^{\circ} \mathrm{F}$ & $-20^{\circ} \mathrm{F}$ to $230^{\circ} \mathrm{F}$ \\
\hline Tolerance to water & Not supplied & Not supplied & Submersible & Submersible \\
\hline Accumulated radiation & Not supplied & Not supplied & Not supplied & Not supplied \\
\hline Arm height at full extension (in.) & Not supplied & Not supplied & Not supplied & Not supplied \\
\hline Arm weight at full extension (in.) & Not supplied & Not supplied & Not supplied & Not supplied \\
\hline Jaw closure force (lb) & Not supplied & Not supplied & 330 & 250 \\
\hline Jaw tong (in.) & 3.5 & Not supplied & Not supplied & Not supplied \\
\hline Wrist torque $(\mathrm{ft} \cdot \mathrm{lbf})$ & 37.5 & Not supplied & Not supplied & Not supplied \\
\hline \multicolumn{5}{|l|}{ Cost Estimates } \\
\hline Manipulator & Not supplied & Not supplied & $\$ 200,000$ & $\$ 200,000$ \\
\hline Controller & Not supplied & Not supplied & $\$ 100,000$ & $\$ 100,000$ \\
\hline Supplier Chose to Review Data & No & No & Yes & Yes \\
\hline
\end{tabular}

a Arm with controller, 


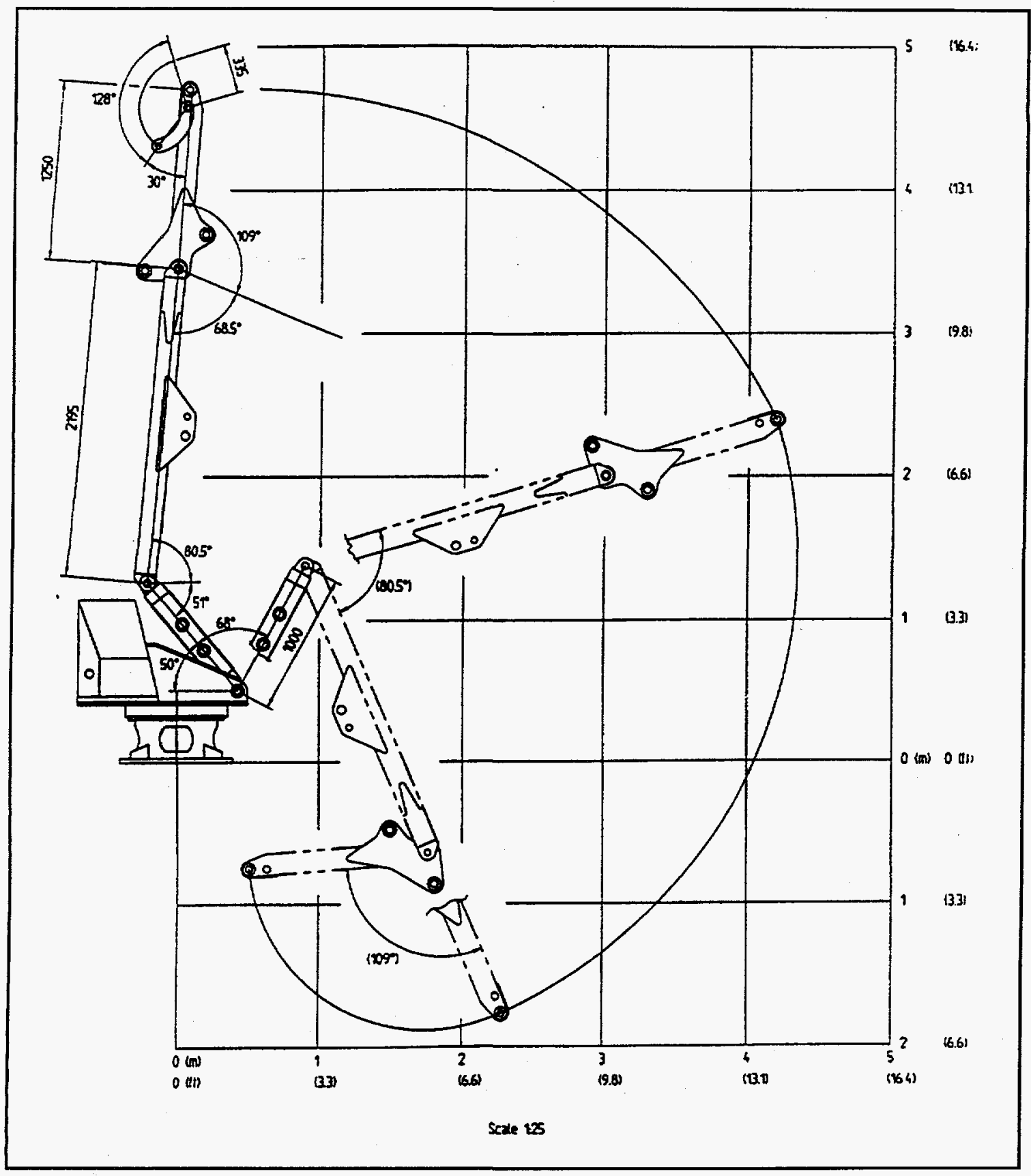

FIGURE 2 BM3000 Manipulator, Manufactured by Brokk, North American Sales, Inc. 


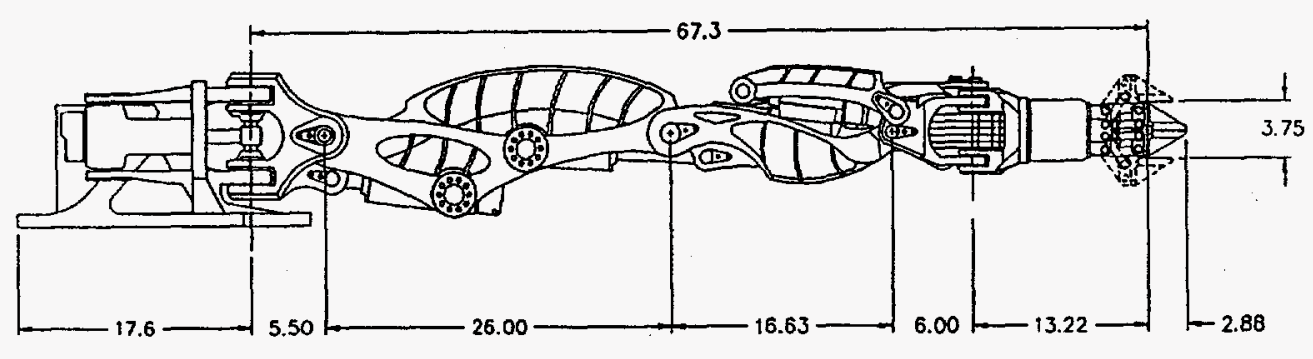

FIGURE 3 Conan T Manipulator, Manufactured by GEC Alsthom Schilling Robotic Systems, Inc.

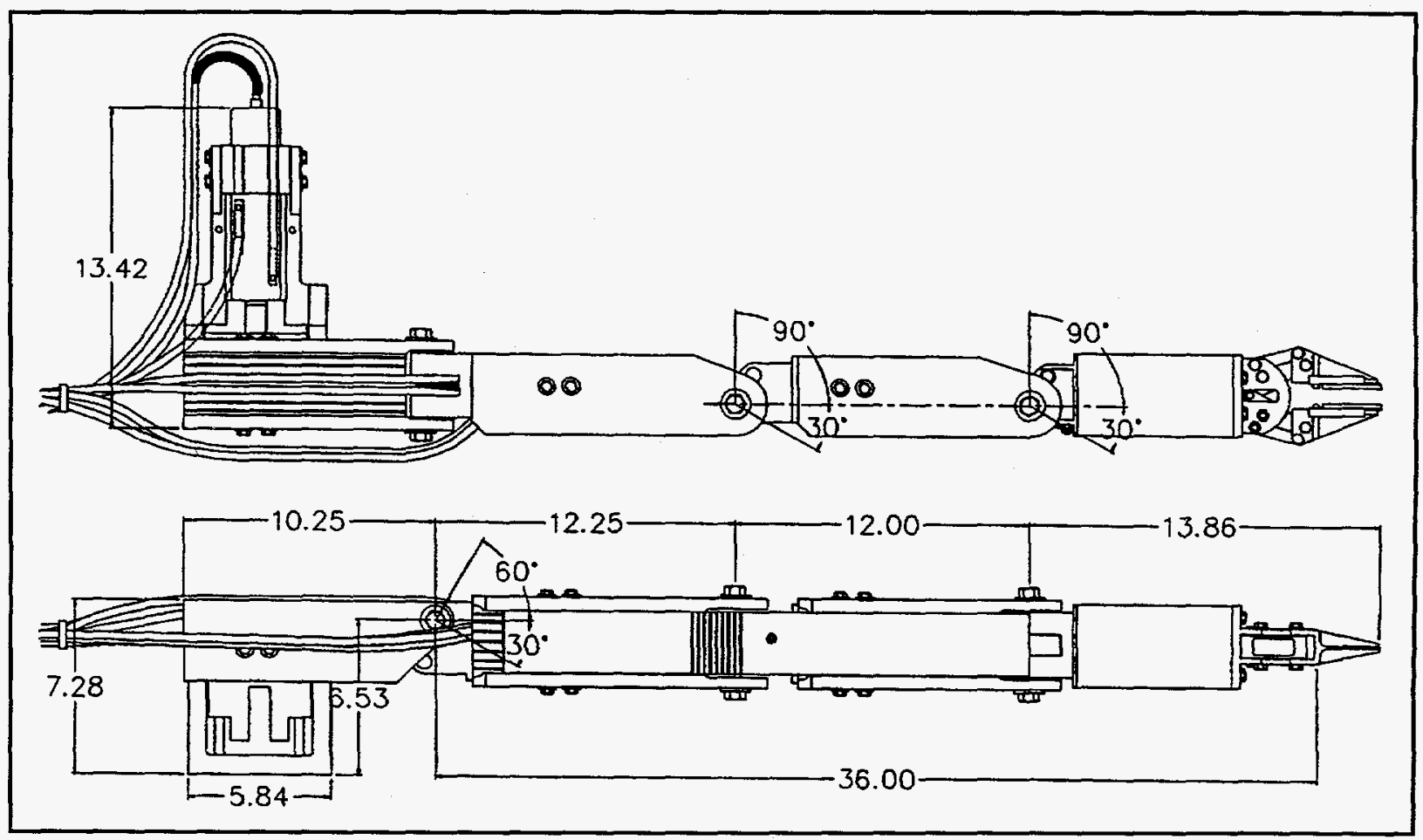

FIGURE 4 Hydra T Manipulator, Manufactured by GEC Alsthom Schilling Robotic Systems, Inc. 


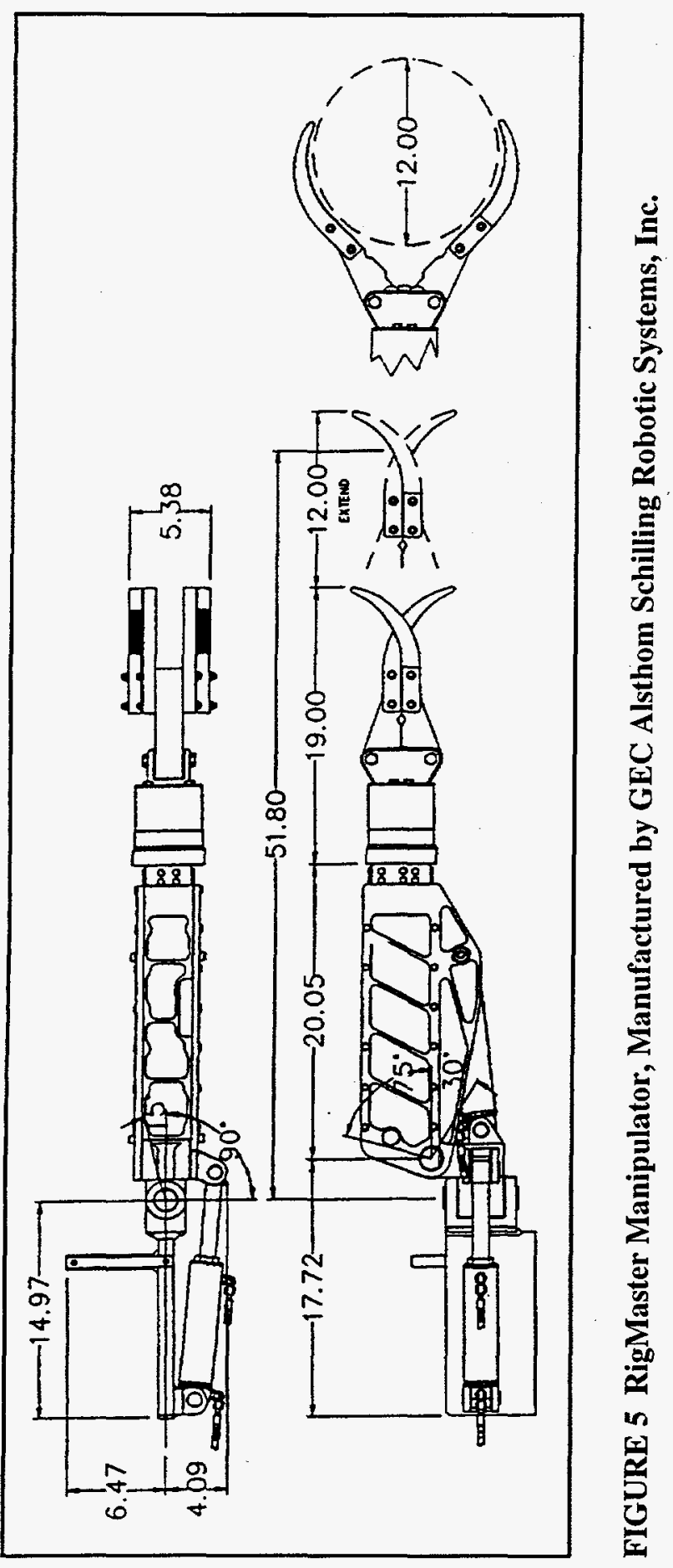



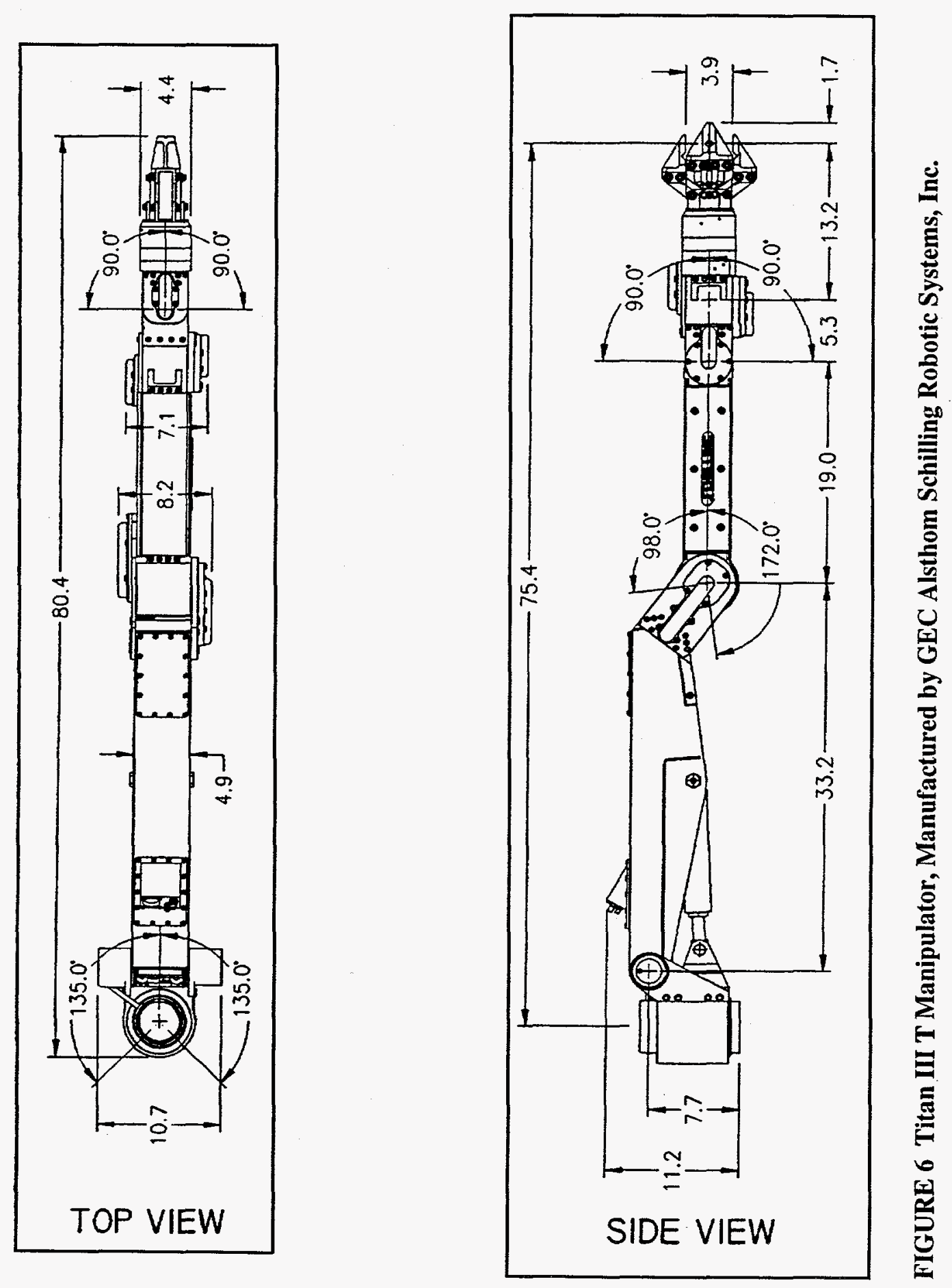
TABLE 3 Category RA2 - Specifications of Hydraulically Powered Manipulators ("Near Commercial")

Supplier and Name of Manipulator

Specification

Weight (lb)

Maximum reach $(\mathrm{ft})$

Degrees of freedom

Maximum payload @ full extension (lb)

Boom

Shoulder azimuth yaw

Shoulder pitch (pivot)

Shoulder roll (rotate)

Elbow pitch (pivot)

Wrist yaw (side pivot)

Wrist pitch (pivot)

Wrist roll (rotate)

Temperature $\left({ }^{\circ} \mathrm{F}\right)$

Tolerance to water

Accumulated radiation

Arm height @ full extension (in.)

Arm width @ full extension (in.)

Jaw closure force (lb)

Jaw tong

Wrist torque (ft・lbf)

\section{Cost Estimates}

Manipulator with controller

Supplier Chose to Review Data
Spar Aerospace Limited

RMS 2500 Series

Spar Aerospace Limited

Modified Light Duty Utility

Arm System (MLDUA)

(see Figure 8)

Arm: 1,500

Total System: 29,500

15.2 to tool plate;

18.2 including end effector;

37 vertical pos. mast reach

7 plus gripper 200

See Figure 8

$\pm 180^{\circ}$

0 to $105^{\circ}$

NA

Elbow yaw (X2) $\pm 100^{\circ}$

$$
\begin{gathered}
\pm 100^{\circ} \\
\pm 90^{\circ} \\
\pm 180^{\circ}
\end{gathered}
$$

Operate 70-90

Storage -31-131

5-90\% humidity

End-effector and wrist -

$1.9 \times 10^{6} \mathrm{rad}$; elbow, shoulder, base $-1.1 \times 10^{5} \mathrm{rad}$

334

80 to 3,200

N/A

Yaw 833; Pitch 2,083

Operate 50-150

Storage -20-150

10-100\% humidity

$2,000 \mathrm{rad} / \mathrm{h}$ up to $10^{8} \mathrm{rad}$

(minimum $10^{6} \mathrm{rad}$ before

maintenance)

See Figure 10

See Figure 10

500

N/A

Roll 58; Pitch

2,000; Yaw 583

Not supplied

Not supplied

Yes 


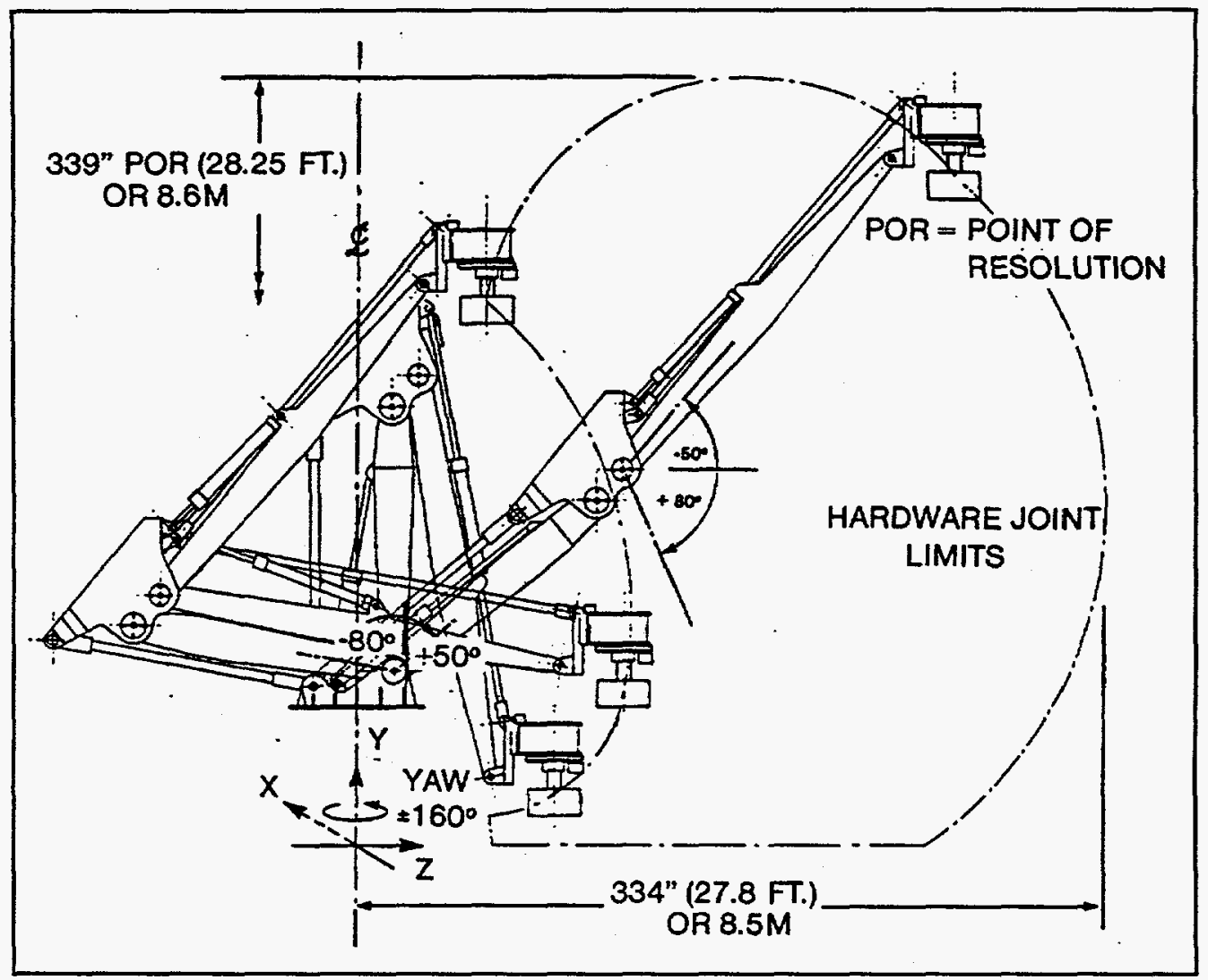

FIGURE 7 RMS 2500 Series Manipulator, Manufactured by Spar Aerospace Limited

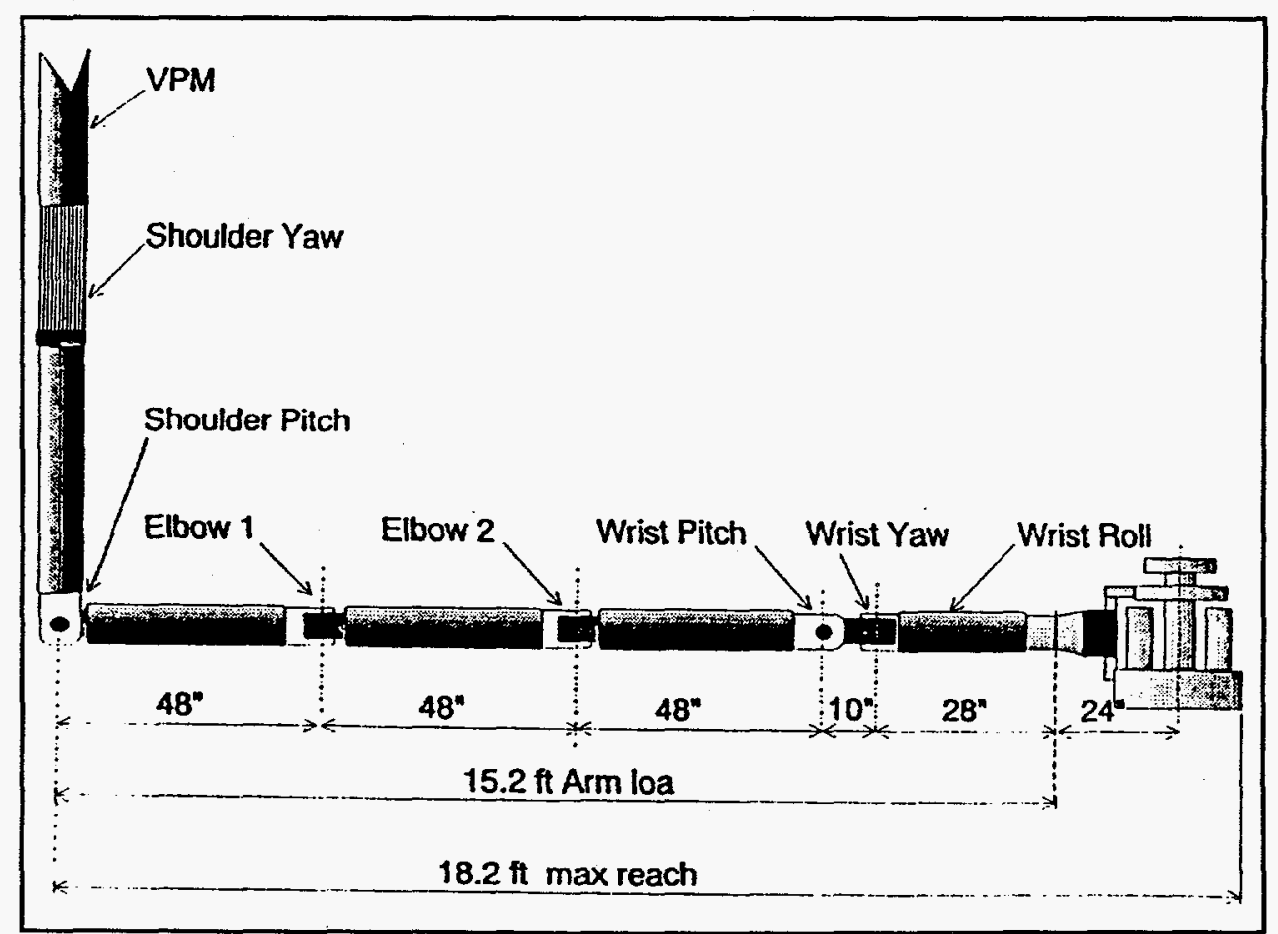

FIGURE 8 MLDUA Manipulator, Manufactured by Spar Aerospace Limited 
TABLE 4 Category RA1 - Specifications of Electrically Powered Manipulators (Commercially Available)

\begin{tabular}{|c|c|c|c|c|}
\hline Specification & \multicolumn{4}{|c|}{ Supplier and Name of Manipulator } \\
\hline Weight (lb) & 31.8 & 72.7 & 268.2 & 218.2 \\
\hline Maximum reach (in.) & 49.2 & 60 & 59.5 & 85.6 \\
\hline Degrees of freedom & 6 plus grip & 6 plus grip & 6 plus grip & 6 plus grip \\
\hline Maximum payload at full extension (lb) & 22 & 62 & 55 & 220 \\
\hline Shoulder azimuth yaw & NA & NA & NA & NA \\
\hline Shoulder pitch (pivot) & $230^{\circ}$ range & $230^{\circ}$ range & $220^{\circ}$ range & $180^{\circ}$ range \\
\hline Shoulder roll (rotate) & $320^{\circ}$ range & $320^{\circ}$ range & $320^{\circ}$ range & $320^{\circ}$ range \\
\hline Elbow pitch (pivot) & $250^{\circ}$ range & $250^{\circ}$ range & $270^{\circ}$ range & $220^{\circ}$ range \\
\hline Wrist yaw (side pivot) & NA & NA & NA & NA \\
\hline Tool roll (rotate) & $540^{\circ}$ range & $540^{\circ}$ range & $532^{\circ}$ range & $360^{\circ}$ range \\
\hline Wrist pitch (pivot) & $240^{\circ}$ range & $240^{\circ}$ range & $200^{\circ}$ range & $220^{\circ}$ range \\
\hline Wrist roll (rotate) & $320^{\circ}$ range & $320^{\circ}$ range & $532^{\circ}$ range & $360^{\circ}$ range \\
\hline Temperature $\left({ }^{\circ} \mathrm{C}\right)$ & $0-50$ & $0-40$ & $5-40$ & $0-50$ \\
\hline Tolerance to water & Non-condensing & Non-condensing & $50 \%$ humidity at $40^{\circ} \mathrm{C}$ & Non-condensing \\
\hline Accumulated radiation (MGy) & 1 & 1 & 1 & 1 \\
\hline Arm height at full extension (in.) & Not supplied & Not supplied & Not supplied & Not supplied \\
\hline Arm width at full extension (in.) & Not supplied & Not supplied & Not supplied & Not supplied \\
\hline Jaw closure force (lb) & Not supplied & Not supplied & Not supplied & Not supplied \\
\hline Jaw tong (in.) & Not supplied & Not supplied & Not supplied & Not supplied \\
\hline Wrist torque & Not supplied & Not supplied & Not supplied & Not supplied \\
\hline \multicolumn{5}{|l|}{ Cost Estimates } \\
\hline Manipulator & Not supplied & Not supplied & Not supplied & Not supplied \\
\hline Controller & Not supplied & Not supplied & Not supplied & Not supplied \\
\hline Supplier Chose to Review Data & No & No & No & No \\
\hline
\end{tabular}


Supplier and Name of Manipulator

\begin{tabular}{|c|c|c|c|c|}
\hline Specification & $\begin{array}{l}\text { GEC Alstrom } \\
\text { Schilling: } \\
\text { Helios } \\
\text { (see Figure 12) }\end{array}$ & $\begin{array}{c}\text { PaR Systems: } \\
3350 \\
\text { (see Figure 9) }\end{array}$ & $\begin{array}{c}\text { PaR Systems: } \\
4350 \\
\text { (see Figure 10) }\end{array}$ & $\begin{array}{c}\text { PaR Systems: } \\
6350 \\
\text { (see Figure 11) }\end{array}$ \\
\hline Weight (lb) & 300 & 350 & 450 & 850 \\
\hline Maximum reach (in.) & 72 & 98 & 112 & 121 \\
\hline Degrees of freedom & 6 plus grip & 6 plus grip & 6 plus grip & 6 plus grip \\
\hline Maximum payload at full extension (lb) & 150 & 150 & 250 & 500 \\
\hline Shoulder azimuth yaw & NA & NA & NA & NA \\
\hline Shoulder pitch (pivot) & $240^{\circ}$ range & $210^{\circ}$ range & $210^{\circ}$ range & $210^{\circ}$ range \\
\hline Shoulder roll (rotate) & $540^{\circ}$ range & $370^{\circ}$ range & $370^{\circ}$ range & $370^{\circ}$ range \\
\hline Elbow pitch (pivot) & $240^{\circ}$ range & $240^{\circ}$ range & $240^{\circ}$ range & $240^{\circ}$ range \\
\hline Wrist yaw (side pivot) & NA & $180^{\circ}$ range & $180^{\circ}$ range & $180^{\circ}$ range \\
\hline Forearm roll (rotate) & $540^{\circ}$ range & NA & NA & NA \\
\hline Wrist pitch (pivot) & $240^{\circ}$ range & $300^{\circ}$ range & $300^{\circ}$ range & $300^{\circ}$ range \\
\hline Wrist roll (rotate) & $360^{\circ}$ continuous & $360^{\circ}$ cont. & $360^{\circ}$ cont. & $360^{\circ}$ continuous \\
\hline Temperature $\left({ }^{\circ} \mathrm{C}\right)$ & 40 & $0-45$ & $0-45$ & $0-45$ \\
\hline Tolerance to water & Underwater $100 \mathrm{ft}$ & $100 \%$ humidity $\max$ & $100 \%$ humidity $\max$ & $100 \%$ humidity maximum \\
\hline Accumulated radiation & $10^{7} \mathrm{rad}$ & $10^{8} \mathrm{rad}$ & $10^{8} \mathrm{rad}$ & $10^{8} \mathrm{rad}$ \\
\hline Arm height at full extension (in.) & 13.5 & 9.25 & 10.5 & 11.5 \\
\hline Arm width at full extension (in.) & 13.5 & 10.25 & 10.25 & 24.5 \\
\hline Jaw closure force (lb) & 200 & $0-500$ & $0-500$ & $0-500$ \\
\hline Jaw tong (in.) & 4 & 5 and 8 & 5 and 8 & 5 and 8 \\
\hline Wrist torque (lbf) & 150 & Not supplied & Not supplied & Not supplied \\
\hline \multicolumn{5}{|l|}{ Cost Estimates } \\
\hline Manipulator & $\$ 300,000^{\mathbf{a}}$ & $\$ 171,000$ & $\$ 182,000$ & $\$ 193,000$ \\
\hline Controller & Not supplied & $\$ 85,000$ & $\$ 85,000$ & $\$ 85,000$ \\
\hline Supplier Chose to Review Data & Yes & Yes & Yes & Yes \\
\hline
\end{tabular}

a Manipulator with controller. 


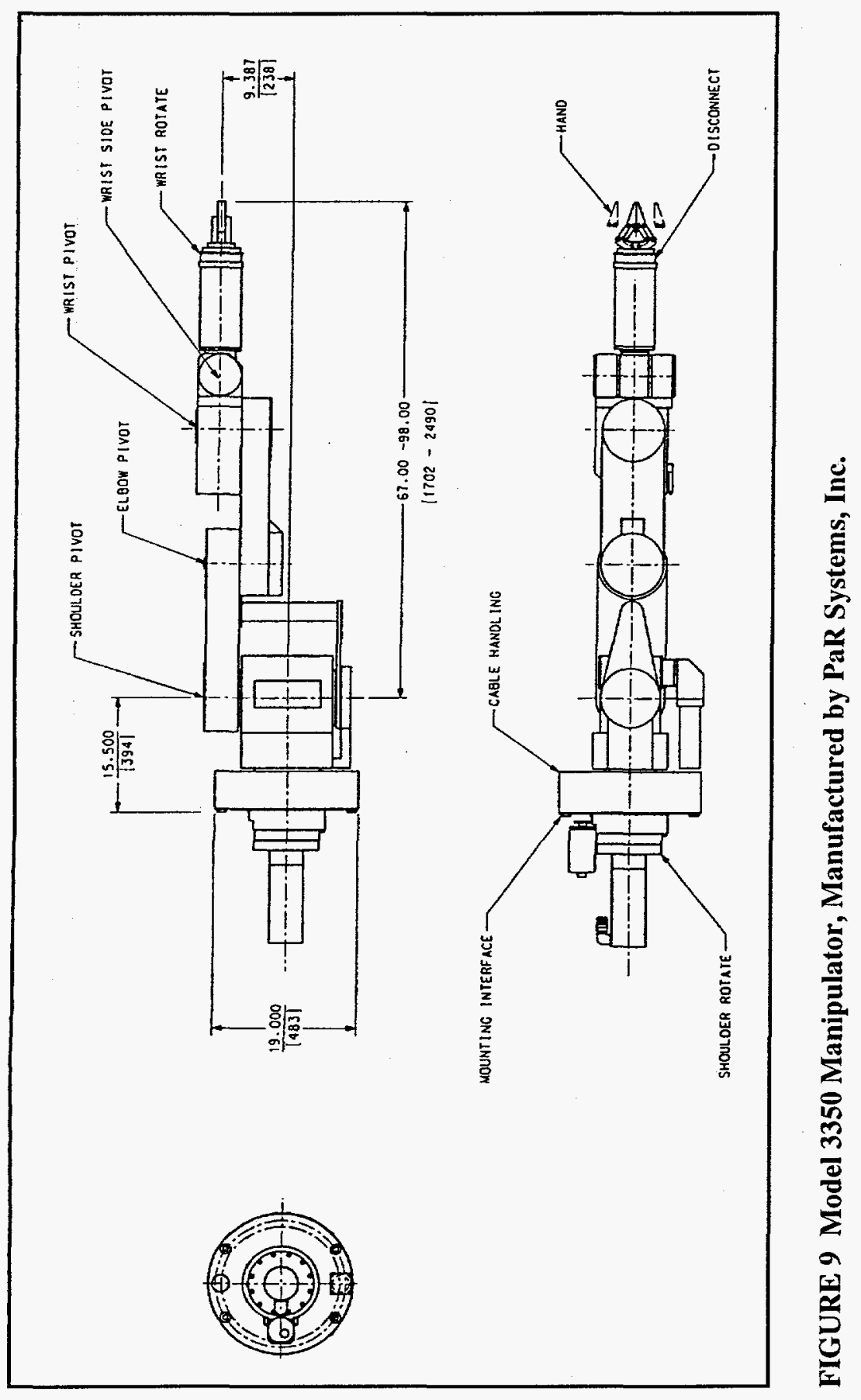




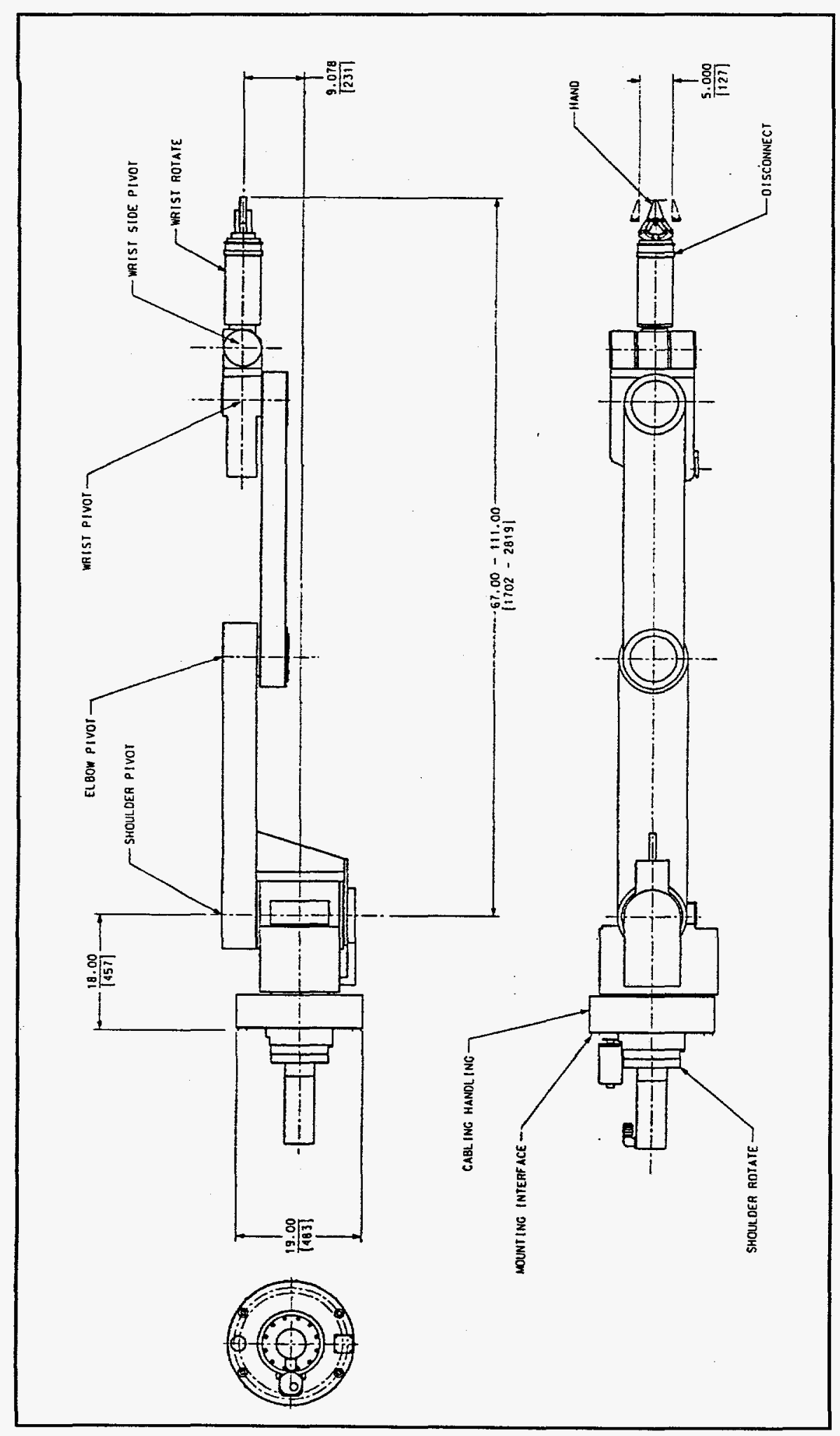

过 


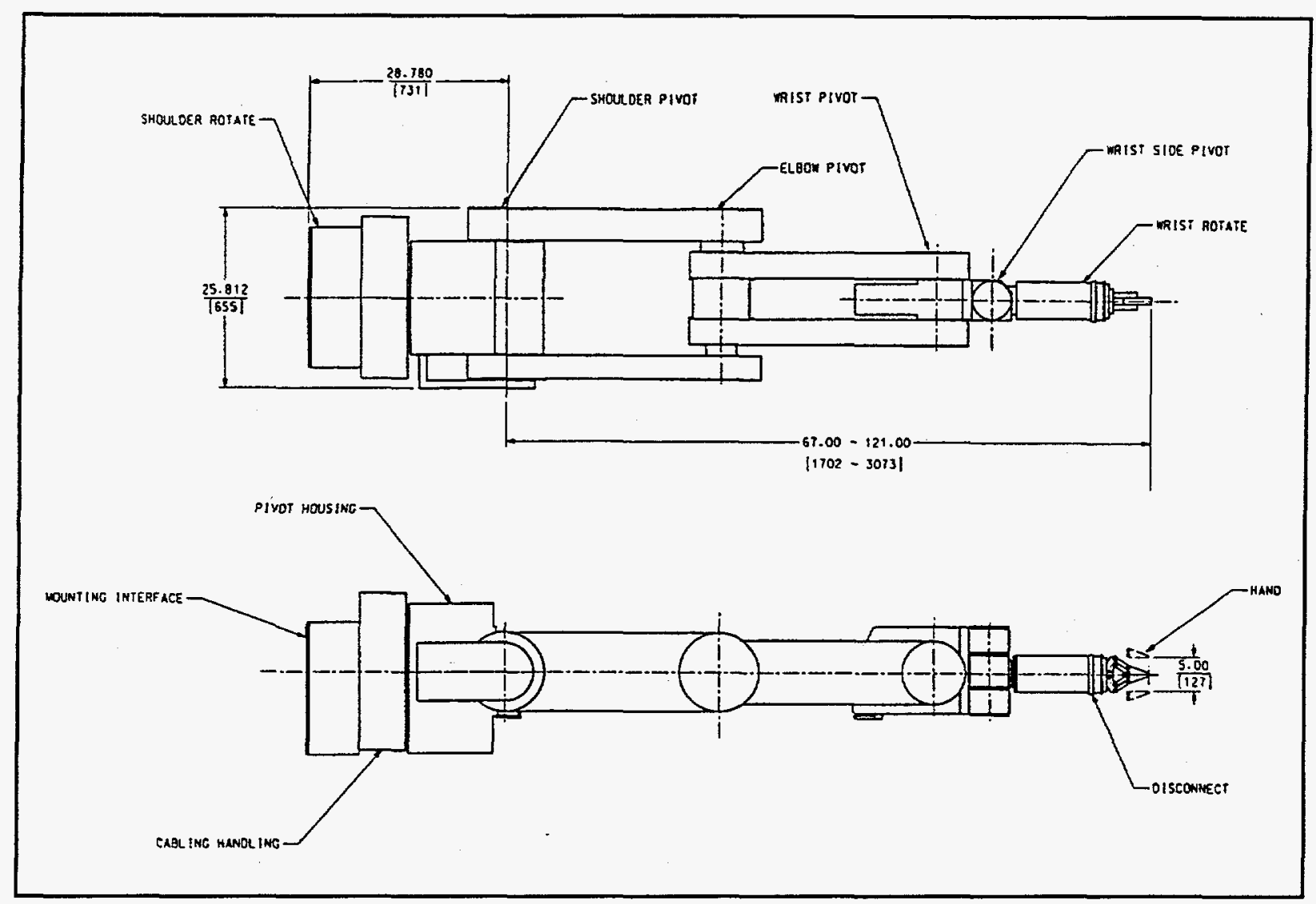

FIGURE 11 Model 6350 Manipulator, Manufactured by PaR Systems, Inc.

end-effectors may have to be reviewed and possibly modified for particular applications. Some endeffectors are wrist-held and others are gripper-held. There are also universal end-effector interfaces for using multiple end-effectors. Table 7 lists the types and costs of end-effectors provided by several suppliers.

\subsubsection{Other End-Effectors}

Special end-effectors or tools (those not listed as standard) have been developed. These endeffectors have been customized for the special applications listed below.

- Autogenous welding (without filler wire)

- Closed circuit television - CCTV (viewing)

- Chisels (pneumatic)

- Electrical discharge machining (EDM) - for metal sampling

- Grinders/abrasive wheels

- Hydraulic torque wrench 


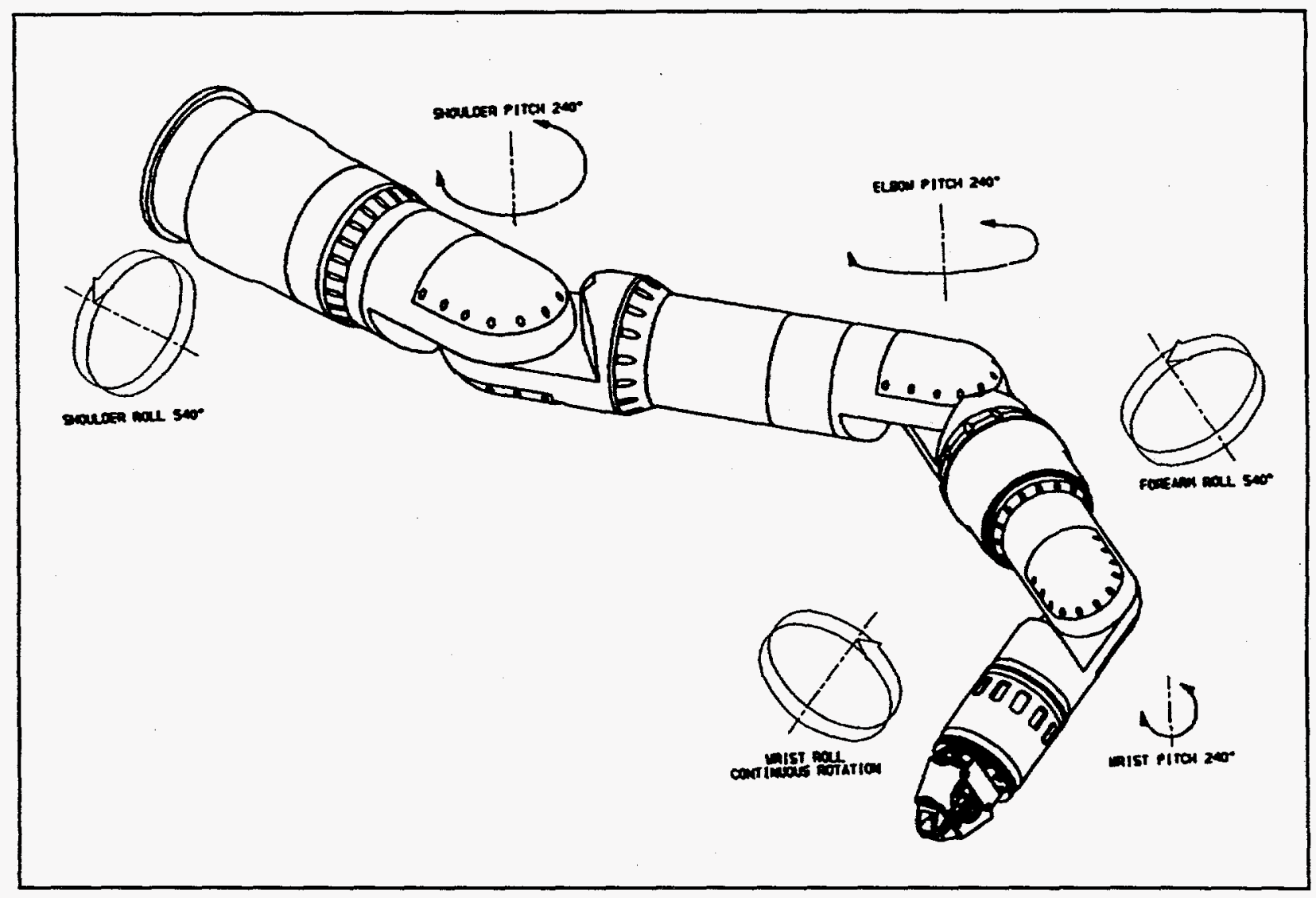

FIGURE 12 Helios Manipulator - Manufactured by GEC Alsthom Schilling Robotic Systems, Inc.

- Liquid penetrant examination (PT)

- Ultrasonic examination (UT)

- Mapping digitizer

- Marking

- Molding

- Oxy-fuel torches

- Plasma torches (underwater or in air with $0^{\circ}-90^{\circ}$ nozzles)

- Pneumatic torque/impact wrenches

- Screwdrivers

- Shears (hydraulic)

- Vacuum cleaning

- Welding (with filler wire) 
TABLE 5 Category RA2 - Specifications of an Electrically Powered Manipulator ("Near Commercial")

Omniview, Inc.

TeleMate Manipulator

Selection

(see Figure 13)

Weight (lb)

92.41

Maximum reach (in.)

43.3

Degrees of freedom

Maximum payload at full extension (lb)

6 plus grip

Shoulder azimuth yaw

33

Shoulder pitch (pivot)

NA

Shoulder roll (rotate)

$\pm 90^{\circ}$

Elbow pitch (pivot)

$\pm 45^{\circ}$

Wrist yaw (side pivot)

$+160^{\circ},-50^{\circ}$

$\pm 180^{\circ}$

Wrist pitch (pivot)

Wrist roll (rotate)

$+45^{\circ},-120^{\circ}$

$\pm 180^{\circ}$

Temperature $\left({ }^{\circ} \mathrm{C}\right)$

100

Tolerance to water

$100 \%$ humidity

Accumulated radiation (rad)

$10^{6}$

Arm height at full extension (in.)

12

Arm width at full extension (in.)

19

Jaw tong (in.)

3.5

Wrist torque (ft・lbf)

2

Cost Estimate

Manipulator with controller

$\$ 500,000$

Supplier Chose to Review Data

Yes 


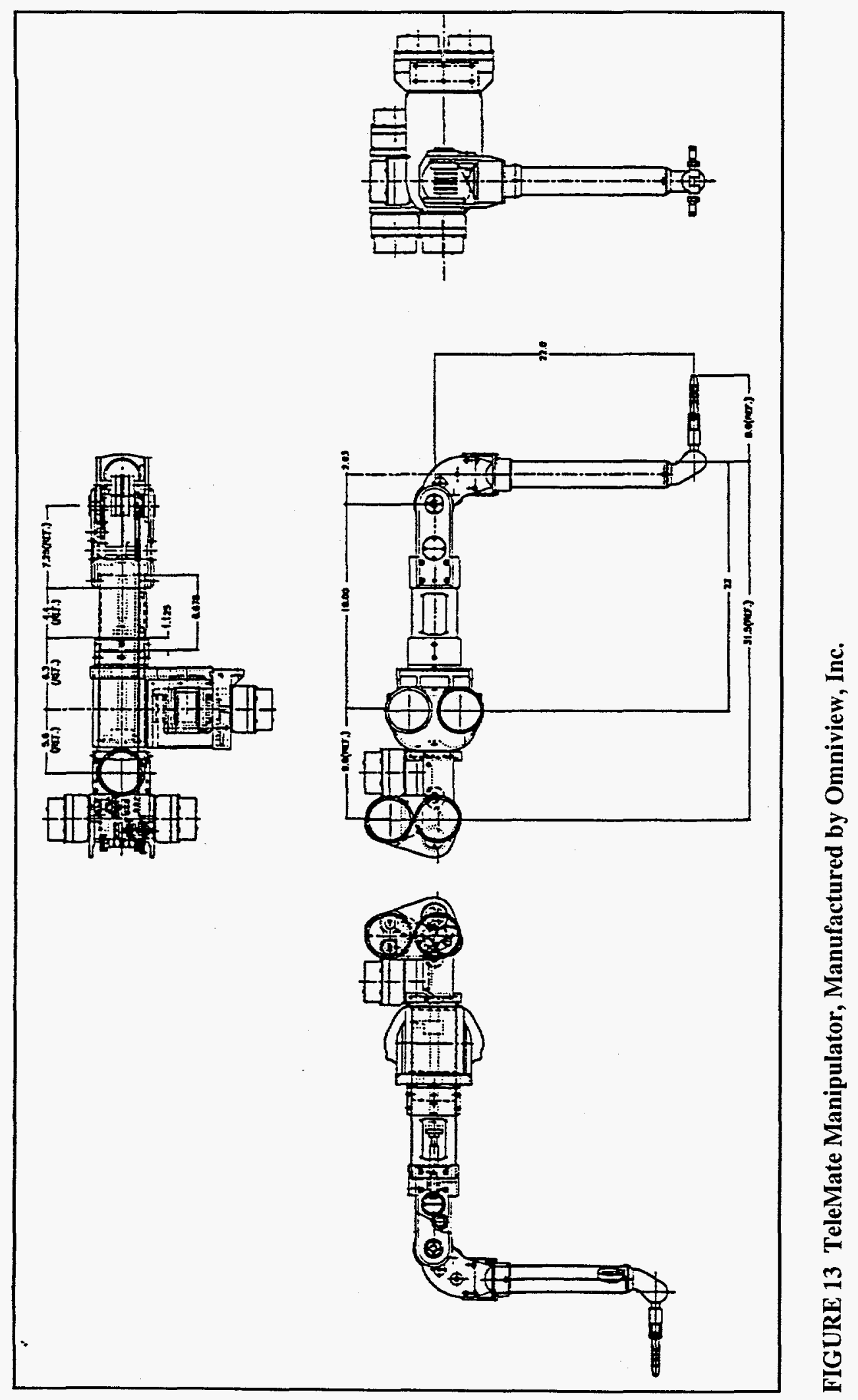




\section{TABLE 6 Features of Controllers}

\begin{tabular}{|c|c|}
\hline Supplier & Features \\
\hline AEA Technology & Industrial robotic controller \\
\hline Brokk & $\begin{array}{l}\text { Handheld control box with joysticks } \\
\text { Hydraulic control valves } \\
\text { Operator always in loop } \\
\text { Visually or remote CCTV viewed }\end{array}$ \\
\hline Deneb Robotics, Inc. & Dedicated arm controller \\
\hline $\begin{array}{l}\text { GEC Alsthom Schilling } \\
\text { Robotic Systems, Inc. }\end{array}$ & $\begin{array}{l}\text { Teach/repeat } \\
\text { Force feedback option } \\
\text { Microprocessor/control station } \\
\text { Remote server driver } \\
\text { Enclosed wiring and embedded hydraulics } \\
\text { Operator-in-loop or programmed motion } \\
\text { Virtual Motion Environment-based operation option } \\
\text { Visually or remote CCTV viewed }\end{array}$ \\
\hline Kraft TeleRobotics & Dedicated arm controller \\
\hline Omniview, Inc. & Teach/repeat \\
\hline PaR Systems, Inc. & $\begin{array}{l}\text { Teach/repeat } \\
\text { Force feedback option } \\
\text { Control console } \\
\text { Operator-in-loop or programmed motion } \\
\text { Modular controller } \\
\text { Remote motor control center } \\
\text { Self-diagnostics } \\
\text { Touch screen option } \\
\text { Visually or remote CCTV viewed }\end{array}$ \\
\hline Sarcos Inc. & Replica master arm \\
\hline Spar Aerospace Limited & $\begin{array}{l}\text { Handheld control box } \\
\text { Hydraulic control valves } \\
\text { Control panel selects modes of manual or computer control } \\
\text { Software for programmed trajectories } \\
\text { Operator-in-loop or programmed motion } \\
\text { Visually or remote CCTV viewed }\end{array}$ \\
\hline $\begin{array}{l}\text { Western Space and } \\
\text { Marine }\end{array}$ & $\begin{array}{l}\text { Force feedback option } \\
\text { Microprocessor/control station } \\
\text { Remote server driver } \\
\text { Operator-in-loop of programmed motion } \\
\text { Visually or remote CCTV viewed }\end{array}$ \\
\hline
\end{tabular}


Cost Estimate

Supplier

Type

(\$)

AEA Technology

Gripper-held:

NEATER jaw (pneumatic gripper)

Not supplied

Power drill (in-line or $90^{\circ}$ )

Not supplied

Vacuum gripper

ARTISAN jaw (hydraulic gripper)

Not supplied

Reciprocating saw

Light-duty cropper (shear)

Not supplied

Not supplied

Not supplied

AECL Technologies

Wrist-held:

Wet replication tool (contaminated metal sampling)

Not supplied

Pressure tube sampling tool

SLAR (spacer location and repositioning) tool

Not supplied

Not supplied

Brokk

Wrist-held:

Hydraulic hammer (boulder-breaking)

22,250

Hydraulic hammer (rock scaling)

22,250

CC400 concrete-crushing jaw

19,250

CC550 concrete-crushing jaw

22,500

GEC Alsthom Schilling

Interface-held:

Robotic Systems, Inc.

Parallel jaw

$13,000^{\mathrm{a}}$

19,500

Claw tool

150,000

Weld location marking tool

6,500

Touch probe

11,000

Air blower

14,500

Heat gun

7,500

Suction tool

9,000

Moisture/instrument probe

10,000

Spray tool

19,000

Rotary power tool, $90^{\circ}$ drill head

12,000

Snips (shear)

7,500

Rotary power tool, in-wire brush

Tool rack

Not supplied

Tool interchange interface

69,000 
TABLE 7 (Cont.)

\begin{tabular}{|c|c|c|}
\hline Supplier & Type & $\begin{array}{c}\text { Cost Estimate } \\
(\$)\end{array}$ \\
\hline PaR Systems, Inc. & $\begin{array}{l}\text { Interface-held: } \\
\text { Plasma torch } \\
\text { Router (8 electric) } \\
\text { Vacuum-collection system } \\
\text { Circular saw } \\
\text { Pipe cutter } \\
\text { Wrist rotate and gripper } \\
\text { Abrasive saw } \\
\text { Grinding tool } \\
\text { Nut splitter } \\
\text { Bolt cutter } \\
\text { Tool rack } \\
\text { EOA quick disconnect }\end{array}$ & $\begin{array}{r}26,000 \\
32,000 \\
18,000 \\
4,800 \\
6,200 \\
14,000 \\
5,700 \\
5,700 \\
5,700 \\
5,700 \\
11,000 \\
4,200\end{array}$ \\
\hline Sarcos, Inc. & $\begin{array}{l}\text { Wrist-held: } \\
\text { Dextrous Hand } \\
\text { (16 degrees of freedom, } 3 \text { fingers, } \\
1 \text { thumb) } \\
\text { Morph Hand (parallel jaw gripper plus } \\
2 \text { degrees of freedom, thumb) }\end{array}$ & $\begin{array}{l}\text { Not supplied } \\
\text { Not supplied }\end{array}$ \\
\hline
\end{tabular}

a Included with standard system.

\subsubsection{Universal End-Effector Interfaces}

Of the suppliers that provide standard end-effectors, three also provide a universal endeffector interface:

- AEA Technology.

- GEC Alsthom Schilling Robotic Systems, Inc.

- PaR Systems, Inc.

Three other companies that provide end-effector (tool) changing systems were found:

- Products for Automation (PFA).

- Robotic Accessories.

- Techno Sommer Automatic. 
A universal end-effector interface may be important in some applications for remotely exchanging tools. The services (electrical, hydraulic, pneumatic, etc.) required for remotely attaching and detaching the end-effector to the arm are integrated with the interface plate. Services for the proper functioning of the end-effector may feed through the interface plate or they may be attached directly to the end-effector. A tool rack is usually required when multiple tools are used. Interface plate connectors may include such services as pneumatic supply/return, hydraulic supply/return, electric power (AC or DC), electric ground, signal output/return, and coaxial cable. Other services can be included for custom applications.

\subsection{DELIVERY SYSTEM INFORMATION}

\subsubsection{Suppliers Contacted and Categories}

As mentioned previously, the focus for delivery systems is on masts, overhead cranes, and gantries. As information was gathered, it became apparent that delivery systems are not as standardized as manipulators. Masts, cranes, and gantries are usually designed and built for a specific task (e.g., lifting capacity, bridge span, travel distance, and head room).

Argonne contacted 32 suppliers listed in Appendix B to determine if they had commercially available cranes or gantries that could be used as delivery systems for manipulators. After the information provided by the suppliers was reviewed, categories that describe the delivery-system industry were developed. The categorization and distribution of suppliers is shown in Table 8 . Several suppliers provide equipment in more than one category. The suppliers for categories CG1 through CG5 are listed below.

\section{CG1 Design and Build: Full Range}

American Crane \& Equipment Corp., Douglassville, PA

Ederer, Inc., Seattle, WA

Harnischfeger, Milwaukee, WI

IMPSA International, Inc., Pittsburgh, PA (Argentina)

Whiting Corporation, Harvey, IL

Zenar, Oak Creek, WI 
TABLE 8 Categorization and Description of Suppliers of Crane/Gantry Equipment

\begin{tabular}{llc}
\hline Category & \multicolumn{1}{c}{ Description } & $\begin{array}{c}\text { Number } \\
\text { of Suppliers }\end{array}$ \\
\hline CG1 & $\begin{array}{l}\text { Design and build cranes or gantries for a full range of lifting } \\
\text { capacities on the basis of experience and industry standards }\end{array}$ & 6 \\
CG2 & $\begin{array}{l}\text { Design and build cranes or gantries up to a 50-ton-lifting capacity } \\
\text { on the basis of experience and industry standards }\end{array}$ & 5 \\
CG3 & $\begin{array}{l}\text { Provide a standard product line of adjustable hydraulic jack } \\
\text { gantries that can be customized }\end{array}$ & 4 \\
CG4 & $\begin{array}{l}\text { Provide a standard product line of special-use cranes, gantries, or } \\
\text { related equipment that can be customized for an application }\end{array}$ & \\
CG5 & $\begin{array}{l}\text { Provide such devices as booms or lifts that are commercially } \\
\text { available }\end{array}$ & 10 \\
\hline
\end{tabular}

CG2 Design and Build: Up to 50 Tons

Abell-Howe, Forest Park, IL

AEA Technology, Pittsburgh, PA (United Kingdom)

The Bartholomew Co., Inc., Westbury, NY

PaR Systems, Inc., Shoreview, MN

Stock Equipment Co., Chagrin Falls, $\mathrm{OH}$

\section{CG3 Hydraulic Jack Gantries}

4 Point Lift Systems, Moline, IL

J \& R Engineering, Big Bend, WI

\section{CG4 Standard Product: Special Use}

B.E. Wallace Products Corp., Frazer, PA

Flow International, Kent, WA 
Mi-Jack, Hazelcrest, IL

Zimmerman Handling Systems, Madison Heights, MI

\section{CG5 Booms and Lifts}

Brokk, Seattle, WA (Sweden)

4 Point Lift Systems, Moline, IL

Grove Worldwide, Shady Grove, PA

J \& R Engineering Company, Inc., Big Bend, WI

Mi-Jack, Hazelcrest, IL

RedZone Robotics, Inc., Pittsburgh, PA

Resource Services International, Cleveland, $\mathrm{OH}$

Western Space and Marine, Santa Barbara, CA

\subsubsection{Delivery System Types}

\subsubsection{Crane/Gantry}

A crane can serve as an overhead X-, Y-, and Z-axes delivery system for a manipulator. One type of crane used for these applications is called a bridge crane. A bridge spans parallel rails and provides the $\mathrm{X}$ and $\mathrm{Y}$ motion for a trolley. A cable hoist or telescoping mast provides $\mathrm{Z}$-axis motion. Lifting capacities can vary from light loads to 800 tons or more. Bridge cranes are usually ordered and designed for specific applications. Lifting capacity, bridge span, runway length, and lift height are specified. Figure 14 shows the configuration of a typical bridge crane.

A gantry may also provide an overhead delivery system for a manipulator, except only the $\mathrm{Y}$ and $\mathrm{Z}$ axes are overhead. The gantry frame rides on rails at grade elevation and provides the $\mathrm{X}$ axis motion. A trolley provides the $\mathrm{Y}$-axis motion, and a cable hoist or telescoping mast provides the Z-axis motion. Because gantries do not have to rely on the strength of the building, lifting capacity can vary from light loads to over 2,000 tons. Gantries are more standardized than cranes, but many are ordered and designed for specific applications. Lifting capacity, frame span, runway length, and lift height are specified. Figure 15 shows the configuration of a typical gantry.

The data for the types of cranes/gantries provided by the suppliers in Categories CG1 through CG4 are presented in Tables 9-12. Cost estimates provided by suppliers for a bridge-crane delivery system measuring $25 \mathrm{ft}$ wide $\times 30 \mathrm{ft}$ long $\times 25 \mathrm{ft}$ high with a 2 ton lifting capacity and a telescoping mast varied between $\$ 400,000$ and $\$ 1,000,000$. A cost estimate provided by a different supplier for a similar gantry delivery system was $\$ 450,000$. 


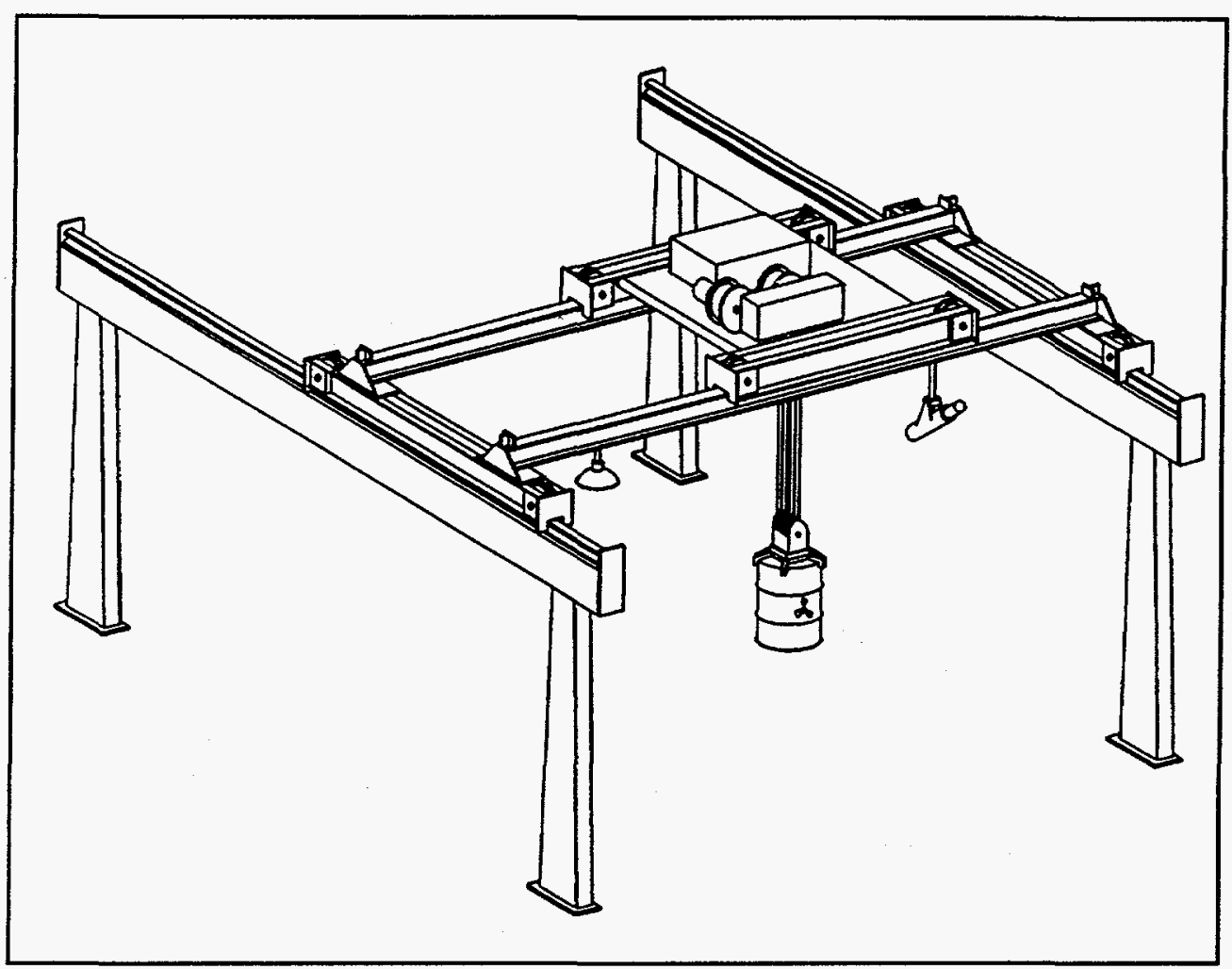

FIGURE 14 Typical Configuration of a Bridge Crane

\subsubsection{Booms and Lifts}

Another option for a manipulator delivery system is booms or boom cranes. Booms are often referred to as "cherry pickers." The main component of a boom is the three- or four-part telescoping mast, which is connected to a vehicle or a platform. Vehicles can be of a rubber-tired or tractor-crawler design. Lifting capacity can be as much as 150 tons, and the extended height can be up to $173 \mathrm{ft}$. Figure 16 shows the typical configuration of a boom. The data for the types of booms and lifts provided by the suppliers in Category CG5 are presented in Table 13.

Lifts are similar to a factory fork-lift truck, but they have much greater capacity and maneuverability. The vehicles are usually of a rubber-tired design. Lifting capacity can be as much as 45 tons, and the extended height can be 30 feet. Figure 17 shows the typical configuration of a lift.

\subsection{SYSTEM INTEGRATION}

Regardless of the delivery system selected, it often has to be integrated with the control of the manipulators. Most delivery system controls are for general positioning, not for the precisely controlled movements required of a manipulator. 


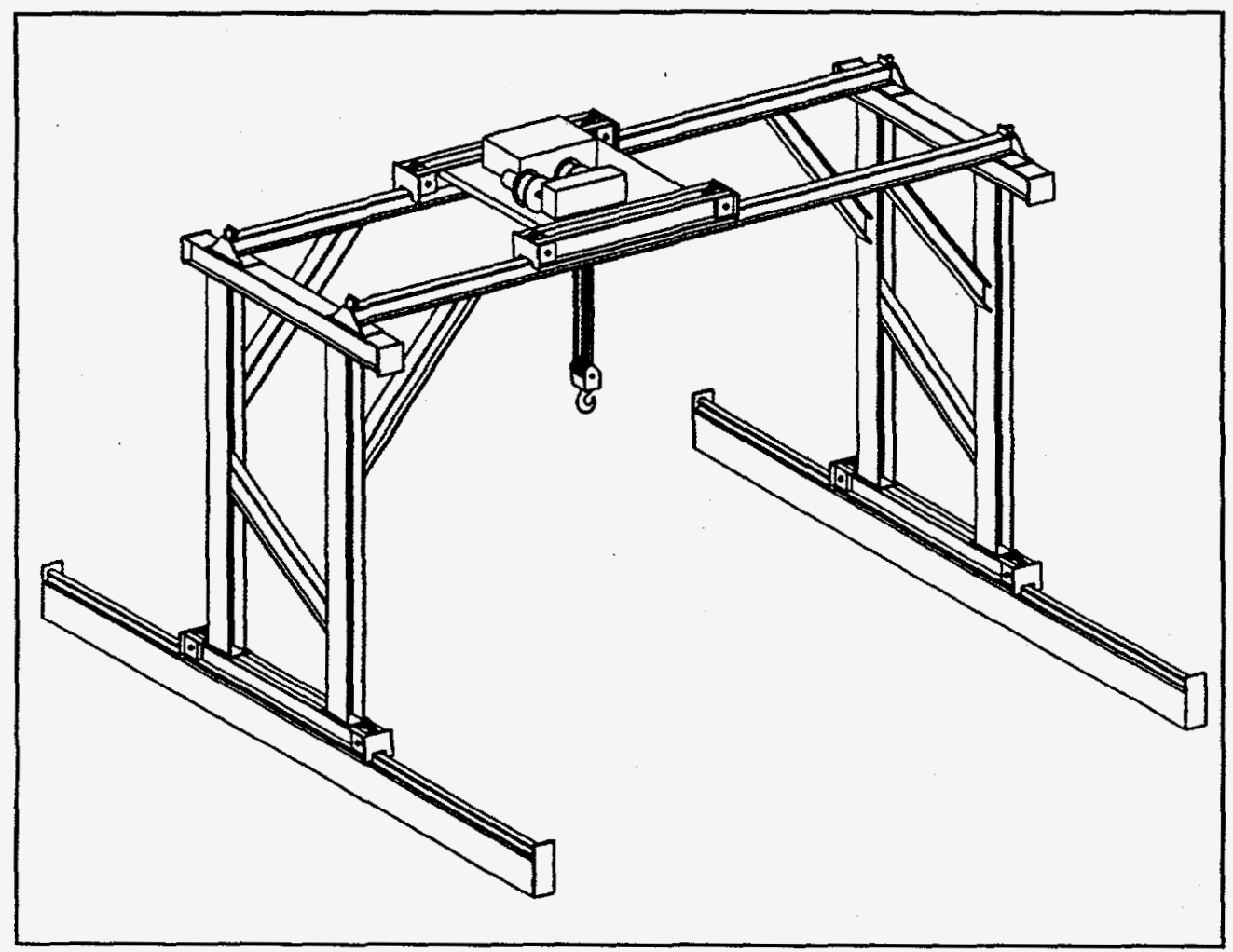

\section{FIGURE 15 Typical Configuration of a Gantry}

This report has grouped special three-axis transport systems often used by robotics manufacturers with industrial cranes and gantries. Construction and performance characteristics may vary greatly. Industrial cranes are usually not designed for precision position control, but they could be modified for a specific application. If a manipulator is attached to the end of a three-axis positioner, the motion of the two devices may have to be coordinated to provide continuous endeffector control. Many applications will not, however, require simultaneous operation of the manipulator and delivery system, thereby simplifying control requirements. Often, as the complexity and sophistication of a robotic system increases, its reliability and availability decrease. For reactor decommissioning activities, reliability is usually more important than finesse. 
TABLE 9 Category CG1 Crane/Gantry Equipment - Design and Build: Full Range

\begin{tabular}{|c|c|c|c|c|c|c|c|c|c|c|c|}
\hline \multirow[b]{2}{*}{ Company } & \multicolumn{3}{|c|}{ Lifting Capacity (ton) } & \multirow{2}{*}{$\begin{array}{l}\text { Bridge } \\
\text { Crane }\end{array}$} & \multirow[b]{2}{*}{ Gantry } & \multirow{2}{*}{$\begin{array}{l}\text { Polar } \\
\text { Crane }\end{array}$} & \multirow{2}{*}{$\begin{array}{c}\text { Mono- } \\
\text { rail } \\
\text { Crane }\end{array}$} & \multirow{2}{*}{$\begin{array}{c}\text { Jib } \\
\text { Crane }\end{array}$} & \multirow{2}{*}{$\begin{array}{l}\text { Telescoping } \\
\text { Mast with } \\
\text { Manipulator } \\
\text { Experience }\end{array}$} & \multirow{2}{*}{$\begin{array}{l}\text { Design for } \\
\text { Nuclear } \\
\text { Decon- } \\
\text { tamination }\end{array}$} & \multirow{2}{*}{$\begin{array}{c}\text { Supplier } \\
\text { Chose to } \\
\text { Review } \\
\text { Data }\end{array}$} \\
\hline & $<50$ & $>50$ & Maximum & & & & & & & & \\
\hline American Crane & Yes & Yes & 200 & Yes & Yes & Yes & Yes & Yes & Yes & Yes & Yes \\
\hline The Bartholomew Co. & Yes & Yes & 200 & Yes & Yes & Yes & Yes & Yes & Yes & Yes & Yes \\
\hline Ederer & Yes & Yes & 700 & Yes & Yes & Yes & Yes & Yes & Yes & Yes & Yes \\
\hline Harnischfeger & Yes & Yes & $N S^{a}$ & Yes & Yes & NS & NS & NS & Yes & No & No \\
\hline IMPSA & Yes & Yes & 750 & Yes & Yes & Yes & No & No & No & No & Yes \\
\hline Whiting & Yes & Yes & 700 & Yes & Yes & Yes & No & No & Yes & No & Yes \\
\hline Zenar & Yes & Yes & 200 & Yes & Yes & No & Yes & Yes & No & No & Yes \\
\hline
\end{tabular}

a NS = not supplied.

TABLE 10 Category CG2 Crane/Gantry Equipment - Design and Build: Up to 50 Tons

\begin{tabular}{|c|c|c|c|c|c|c|c|c|}
\hline Company & $\begin{array}{l}\text { Bridge } \\
\text { Crane }\end{array}$ & Gantry & $\begin{array}{l}\text { Polar } \\
\text { Crane }\end{array}$ & $\begin{array}{l}\text { Mono- } \\
\text { rail } \\
\text { Crane }\end{array}$ & $\begin{array}{c}\text { Jib } \\
\text { Crane }\end{array}$ & $\begin{array}{l}\text { Telescoping } \\
\text { Mast with } \\
\text { Manipulator } \\
\text { Experience }\end{array}$ & $\begin{array}{l}\text { Design for } \\
\text { Nuclear } \\
\text { Decon- } \\
\text { tamination }\end{array}$ & $\begin{array}{c}\text { Supplier } \\
\text { Chose to } \\
\text { Review } \\
\text { Data }\end{array}$ \\
\hline Abell-Howe & Yes & Yes & No & Yes & Yes & $\mathrm{No}^{\mathrm{c}}$ & No & Yes \\
\hline AEA Technology & Yes & $\mathrm{Yes}^{\mathrm{a}}$ & No & No & No & Yes & Yes & No \\
\hline PaR Systems & $\mathrm{Yes}^{\mathrm{a}}$ & Yes & Yes & Yes & No & Yes & Yes & Yes \\
\hline Stock Equipment & Yes $^{b}$ & No & No & No & No & No & Yes & Yes \\
\hline
\end{tabular}

a Supplier refers to these as robotic gantries, but they are essentially gantries.

b Only designed for grappling drums and canisters.

c Produces telescoping mast but has no experience with manipulators. 
TABLE 11 Category CG3 Crane/Gantry Equipment - Hydraulic Jack Gantries

\begin{tabular}{|c|c|c|c|c|c|c|c|c|c|c|}
\hline \multirow[b]{2}{*}{ Company } & \multicolumn{3}{|c|}{ Lifting Capacity (ton) } & \multirow{2}{*}{$\begin{array}{l}\text { Bridge } \\
\text { Crane }\end{array}$} & \multirow[b]{2}{*}{ Gantry } & \multirow{2}{*}{$\begin{array}{l}\text { Polar } \\
\text { Crane }\end{array}$} & \multirow{2}{*}{$\begin{array}{l}\text { Mono- } \\
\text { rail Crane }\end{array}$} & \multirow{2}{*}{$\begin{array}{c}\text { Jib } \\
\text { Crane }\end{array}$} & \multirow{2}{*}{$\begin{array}{l}\text { Telescoping } \\
\text { Mast with } \\
\text { Manipulator } \\
\text { Experience }\end{array}$} & \multirow{2}{*}{$\begin{array}{l}\text { Design for } \\
\text { Nuclear } \\
\text { Decon- } \\
\text { tamination }\end{array}$} \\
\hline & $<50$ & $>50$ & Maximum & & & & & & & \\
\hline 4 Point Lift System & Yes & Yes & 2,000 & No & Yes & No & No & No & No & No \\
\hline J \& R Engineering & Yes & Yes & 900 & No & Yes & No & No & No & No & Yes \\
\hline
\end{tabular}

TABLE 12 Category CG4 Crane/Gantry Equipment - Standard Product: Special Use ${ }^{\text {a }}$

\begin{tabular}{|c|c|c|c|c|c|c|c|c|c|c|}
\hline \multirow[b]{2}{*}{ Supplier } & \multicolumn{3}{|c|}{ Lifting Capacity (ton) } & \multirow{2}{*}{$\begin{array}{l}\text { Bridge } \\
\text { Crane }\end{array}$} & \multirow[b]{2}{*}{ Gantry } & \multirow{2}{*}{$\begin{array}{l}\text { Polar } \\
\text { Crane }\end{array}$} & \multirow{2}{*}{$\begin{array}{l}\text { Mono- } \\
\text { rail Crane }\end{array}$} & \multirow{2}{*}{$\begin{array}{c}\text { Jib } \\
\text { Crane }\end{array}$} & \multirow{2}{*}{$\begin{array}{l}\text { Telescoping } \\
\text { Mast with } \\
\text { Manipulator } \\
\text { Experience }\end{array}$} & \multirow{2}{*}{$\begin{array}{l}\text { Design for } \\
\text { Nuclear } \\
\text { Decon- } \\
\text { tamination }\end{array}$} \\
\hline & $<50$ & $>50$ & Maximum & & & & & & & \\
\hline B.E. Wallace & Yes & No & No supplied & No & $\mathrm{Yes}^{\mathrm{c}}$ & No & No & Yes $^{\mathrm{c}}$ & No & No \\
\hline Flow & Yes & No & Not supplied & Yes $^{b}$ & No & No & No & No & No & No \\
\hline Mi-Jack & Yes & Yes & 75 & No & Yes $^{d}$ & No & No & No & No & No \\
\hline Zimmerman & Yes & No & Not supplied & No & No & No & No & $\mathrm{No}^{\mathrm{e}}$ & No & No \\
\hline
\end{tabular}

\footnotetext{
a These Standard Products can often be modified or customized.

b Used for positioning cutting apparatus and not for lifting.

c Manually powered and operated.

d Provide rubber-tired gantries only.

e Provide cable/drum hoists only.
} 


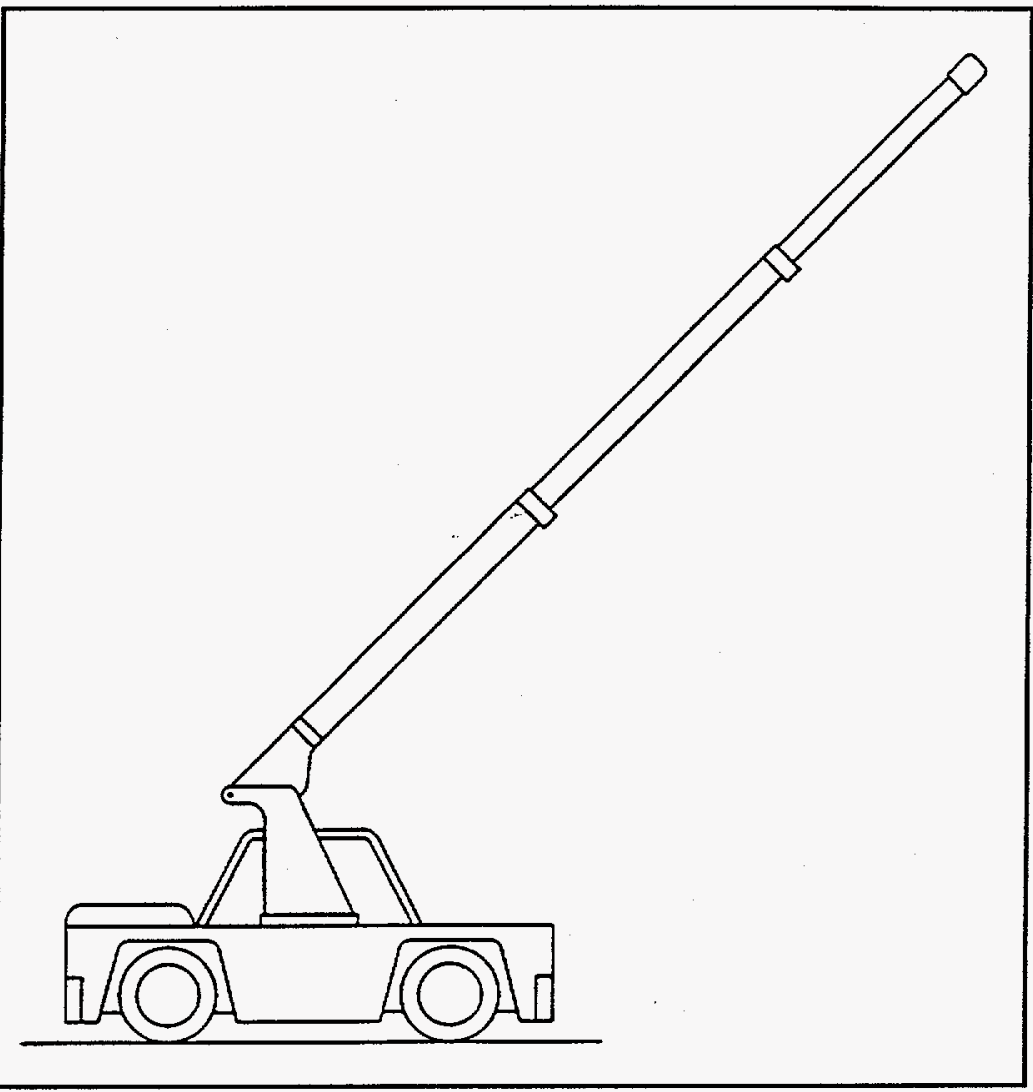

FIGURE 16 Typical Configuration of a Boom

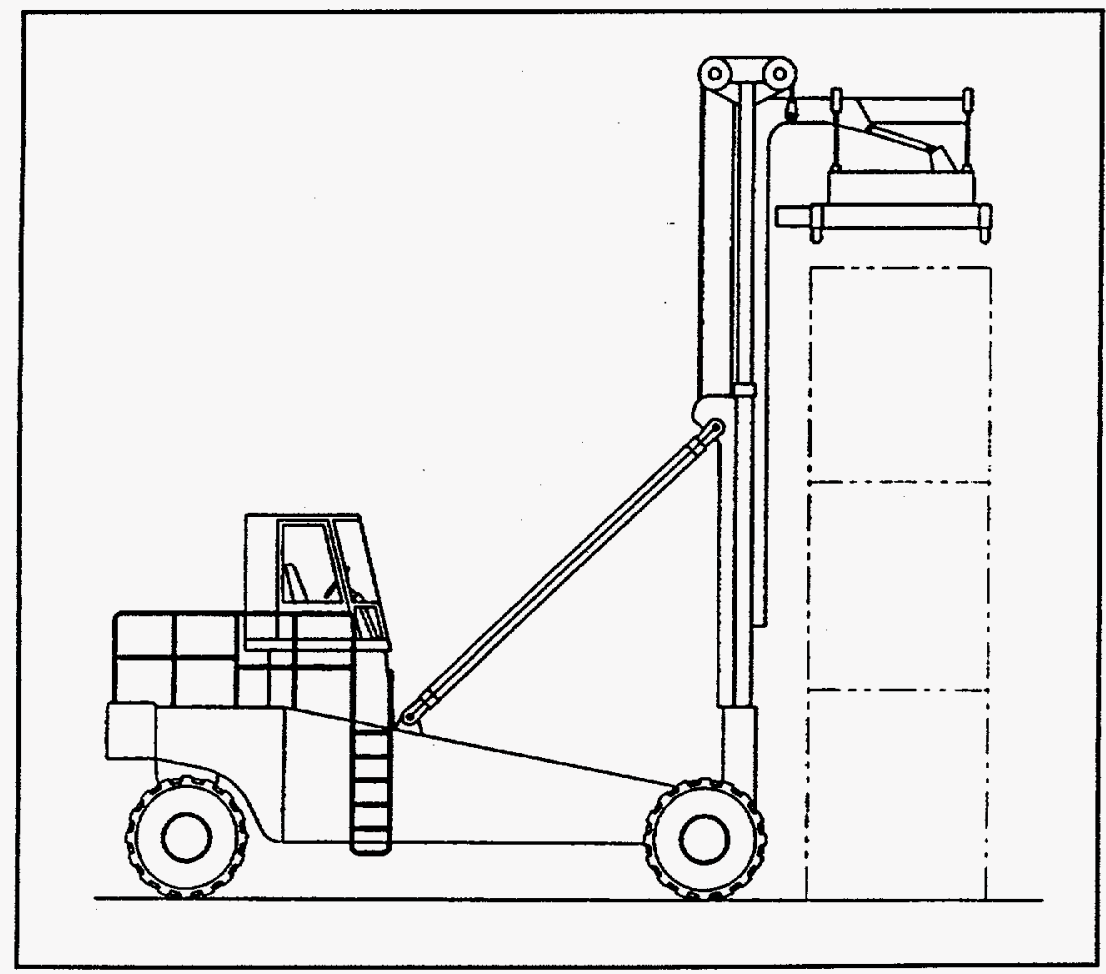

FIGURE 17 Typical Configuration of a Lift 
TABLE 13 Category CG5 Crane/Gantry Equipment - Booms and Lifts

\begin{tabular}{|c|c|c|c|c|c|}
\hline Company & $\begin{array}{c}\text { Maximum }^{\mathrm{a}} \\
\text { Lifting Capacity } \\
\text { (tons) }\end{array}$ & $\begin{array}{c}\text { Maximum } \\
\text { Boom Length } \\
\text { (ft) } \\
\end{array}$ & Boom & Lift & $\begin{array}{c}\text { Maximum } \\
\text { Lift Height } \\
(\mathrm{ft})\end{array}$ \\
\hline Brokk & 0.85 & 13 & $--{ }^{b}$ & $--^{b}$ & 16 \\
\hline 4 Point Lift Systems & 75 & 45 & Yes & No & $N A^{d}$ \\
\hline Grove Worldwide & 150 & 173 & Yes & No & NA \\
\hline J\&R Engineering Co. & 60 & 25 & $--^{b}$ & $--^{b}$ & 34 \\
\hline MI-JACK & 45 & NA & No & Yes & 30 \\
\hline RedZone Robotics, Inc. & $1^{\mathrm{c}}$ & 20 & Yes & No & NA \\
\hline Resource Services Int'l. & $1.5^{\mathrm{c}}$ & 30 & $-\mathrm{-}^{\mathrm{b}}$ & $--^{b}$ & 8 \\
\hline Western Space and Marine & 10 & 24 & $--^{b}$ & $--^{b}$ & 30 \\
\hline
\end{tabular}
a Not at full extension.
b This is a boom and lift combination.
c At full extension.
d Not Applicable. 


\section{BIBLIOGRAPHY}

1. A Compendium of Robotic Equipment Used in Hazardous Environments, 1990, Electric Power Research Institute, Charlotte, NC.

2. Nuclear News Buyers Guide, 1995, American Nuclear Society 38(4), LaGrange Park, IL, March.

3. Nuclear Plant Journal Product \& Services Directory - 1995 Issue, 1994, EQES, Inc., 12(6), Glen Ellyn, IL, November.

4. Tosunoglu, S., 1994, Robotics Technology Assessment for Decontamination and Dismantlement Tasks, U.S. Department of Energy, Office of Technology Development, Robotics Technology Development Program, University of Texas at Austin, Austin, TX, August. 
APPENDIX A:

MANIPULATOR SUPPLIERS 
APPENDIX A: MANIPULATOR SUPPLIERS

TABLE A.1 Categories Used to Describe Robotic Arms

\begin{tabular}{|l|l|}
\hline Category & \multicolumn{1}{|c|}{ Description } \\
\hline RA1 & Commercially available manipulators w/controls \\
\hline RA2 & Provided a manipulator several times ("near commercial") \\
\hline RA3 & Develop unique manipulators for an application \\
\hline RA4 & Purchase manipulators and package them for an application \\
\hline RA5 & Provide crawlers or ROVs \\
\hline RA6 & Provide manipulator controls only \\
\hline RA7 & None of the above \\
\hline
\end{tabular}

Categories are included in the suppliers listed in this appendix.

AEA Technology

241 Curry Hollow Road

Pittsburgh, PA 15236-4696

Contact: Tim Boorman

(412) 655-1200

FAX: (412) 655-2928

Category: RA1

AECL Technologies

9210 Corporate Blvd.

Rockville, MD 20850

Contact: Phil Campbell or Dave

McDougall

(800) 872-2325

FAX: (301) 417-0746

Category: RA3, RA5
Applied Radiological Control (ARC)

500 Chastain Center Blvd.

Kennesaw, GA 30144-59999

Contact: Ted Martucci

(800) 241-6575

FAX: (404) 428-3090

Category: RA7

ARD Environmental Corporation

9151 Rumsey Road

Columbia, MD 21045-1929

Contact: Mark Thomas

(800) 241-6575

FAX: (410) 997-3902

Category: RA5 
ASI Robotic Systems

1250 Crooks Road

Clawson, MI 48017

(313) 288-5070

FAX: (313) 435-3525

Category: RA3

B\&W Nuclear Technologies

P.O. Box 10935

Lynchburg, VA 24506-0935

Contact: Dennis Williams

(800) 231-3628

FAX: (804) 932-2621

Category: RA7

The Bartholomew Co., Inc.

91 New York Ave.

Westbury, NY 11590

Contact: Harry G. Gotte III

(516) 333-0100

FAX: (516) 333-0301

Category: RA4

Benthos, Inc.

49 Edgerton Drive

North Falmouth, MA 02556

Contact: Kevin McCarthy

(800) 446-1222

FAX: (508) 563-6444

Category: RA5

BNFL, Inc.

1835 Terminal Drive, Suite 220

Richland, WA 99352

Contact: Maurice Bullock

(509) 946-4006

FAX: (509) 946-4852

Category: RA1
Brokk, North American Sales, Inc.

1144 Village Way

Monroe, WA 98272

Contact: Mike Martin

(800) 621-7856

FAX: (206) 487-2963

Category: RA1, RA5

Brooks Support Systems Inc.

6546 Pound Road

Williamson, NY 14589

Contact: Frank Dickey

(800) 836-0285

FAX: (315) 589-4089

Category: RA7

Central Research Laboratories

250 Hwy. 19

Red Wing, MN 55066

Contact: Laurie Brown

(612) $385-2142$

FAX: (612) 388-1232

Category: RA7

Cybermotion, Inc.

115 Sheraton Drive

Salem, VA 24153-3003

Contact: Celeste DeCorte

(703) 562-7626

FAX: (703) 562-7632

Category: RA5

Deepsea Power \& Light Co.

4819 Ronson $\mathrm{Ct}$.

San Diego, CA 92111

Contact: Bob Novak

(619) 576-1261

FAX: (619) 576-0219

Category: RA7 
Deneb Robotics, Inc.

3285 Lapeer Road West

P.O. Box 214687

Auburn Hills, MI 48321-4687

Contact: Brian Christenson

(810) 377-6900

FAX: (810) 377-8125

Category: RA6

EPRI

1300 Harris Blvd.

Charlotte, NC 28221

Contact: Ken Brittain

(704) 547-6139

FAX: (704) 547-6168

Category: RA7

Foster-Miller, Inc.

350 Second Ave.

Waltham, MA 02154-1196

Contact: Michael Reinhart

(617) 890-3200

FAX: (617) 890-8515

Category: RA5

GE Nuclear Energy

175 Curtner Ave.

San Jose, CA 95125

Contact: Steve Barber

(408) 925-1151

FAX: (408) 925-1148

Category: RA7

GEC Alsthom Schilling Robotic

Systems, Inc.

1632 DaVinci Court

Davis, CA 95616

Contact: Wes Gerriets or Tyler Schilling

(916) 753-6718

FAX: (916) 753-8092

Category: RA1
General Atomics

P.O. Box 85608

San Diego, CA 92186-9784

Contact: Jim McNair

(619) 455-2802

FAX: (619) 455-2110

Category: RA4

Grove Worldwide

1565 Buchanan Trail East

P.O. Box 21

Shady Grove, PA 17256-0021

Contact: Traci Hahn

(717) $597-8121$

FAX: (717) 597-4062

Category: RA5

Kraft TeleRobotics

11667 West 90th Street

Overland Park, KS 66214

Contact: Steve Harbur

(913) 894-9022

FAX: (913) 894-1363

Category: RA1, RA5

NAC International

655 Engineering Drive

Norcross, GA 30092-2813

Contact: Gary Tjersland

(800) 241-0507

FAX: (404) 447-1797

Category: RA4

NES, Inc.

44 Shelter Rock Road

Danbury, CT 06810-7095

Contact: Jim Hedtke

(203) 796-5273

FAX: (203) 796-3168

Category: RA4 
NSS Numanco, Inc.

West Market Street

Campbelltown, PA 17010

Contact: Fred Erskine

(800) 338-7333

FAX: (717) 838-8400

Category: RA7

Nuclear Systems Associates (NSA)

2741 Saturn Street

Brea, CA 92621

Contact: . Bill Thompson

(714) $996-4670$

FAX: (714) 996-4712

Category: RA3

NUS

910 Clopper Road

Gaithersburg, MD 20878-1399

Contact: Frank Gallo

(800) 368-2755

FAX: (301) 258-8764

Category: RA4

Oceaneering Technologies

501 Prince George Blvd.

Upper Marlboro, MD 20772

Contact: Andrew Resnick

(301) $249-3300$

FAX: (301) 249-4022

Category: RA4

Odetics

1515 South Manchester

Anaheim, CA 92802-2907

Contact: Thomas Bartholet

(714) $780-7805$

FAX: (714) 780-7857

Category: RA3
Omniview, Inc.

7325 Oak Ridge Highway

Knoxville, TN 37921

Contact: Brad Hobbs or Dan Kuban

(423) 690-5600

FAX: (423) 690-2913

Category: RA2

PaR Systems

899 Highway 96 West

Shoreview, MN 55126

Contact: Albert J. Sturm, Jr.

(612) 484-7261

FAX: (612) 483-2689

Category: RA1

Pentek Decontamination Products Div.

1026 Fourth Ave.

Coraopolis, PA 15108

Contact: Eric Crivella

(412) 262-0725

FAX: (412) 262-0731

Category: RA7

Products for Automation (PFA)

15885 W. Overland Drive

New Berlin, WI 53151

Contact: Julie A. Hoaglan

(414) $785-1869$

FAX: (414) 785-9161

Category: RA7

PLS International

10910 Briggs Road

Cleveland, $\mathrm{OH} 44111$

Contact: Dale Vandervort

(216) $252-7700$

FAX: (216) 252-7792

Category: RA4 
RedZone Robotics, Inc.

2425 Liberty Avenue

Pittsburgh, PA 15222-4639

Contact: Leona Bares

(412) 765-3064

FAX: (412) 765-3069

Category: RA4

Resource Services International 6001 Breakwater Avenue

Cleveland, $\mathrm{OH} 44102$

Contact: Harold C. Pedersen

(216) 651-2550

FAX: (216) 651-6811

Category: RA4

Reinhart \& Associates

P.O. Box 9802

Austin, TX 78766

Contact: Louis Strange

(800) 553-5498

FAX: (512) 834-1266

Category: RA7

Remote Systems Services

1800 Freeway Blvd.

Minneapolis, MN 55430-1747

Contact: Glenn McKeag

(612) 484-7261

FAX: (612) 561-4827

Category: RA7

Remotec

114 Union Valley Road

Oak Ridge, TN 37830

Contact: Brad Callahan

(423) 483-0228

FAX: (423) 483-1426

Category: RA5
Robotic Accessories

6555 State Route 202

Tripp City, OH 45371

Contact: Anjela Janowiecki

(513) 667-5705

FAX: (513) 667-7602

Category: RA7

R.O.V. Technologies

P.O. Box 10

Georges Mill, Rte. 142

Vernon, VT 05354-0010

Contact: Troy Dennen

(802) 254-9353

FAX: (802) 254-9354

Category: RA5

Rust Utility Services

P.O. Box 699

110 Plains Road

Essex, CT 06426

Contact: John Allanach

(800) 872-6827

FAX: (203) 767-0612

Category: RA4

Sarcos Inc.

360 Warkara Way

Salt Lake City, UT 84108

Contact: Dave Salmon

(801) 581-0155

FAX: (801) 581-1151

Category: RA1

Silma

1601 Saratoga-Sunnyvale Rd.

Cupertino, CA 95014

Contact: Lisa Lambro

(800) 347-4562

FAX: (408) 725-8955

Category: RA6 
Spar Aerospace Limited

30 Tower Lane

Avon Park South

Avon, CT 06001

Contact: Peter Kruse

(203) 674-1295

FAX: (203) 678-1862

Category: RA2, RA5

Stenning Instruments

604 Via Del Cerro

Camarillo, CA 93010-8439

Contact: Lola Stenning

(805) 484-4996

FAX: (805) 484-5337

Category: RA7

Stock Equipment Co. 16490 Cillicothe Rd.

Chagrin Falls, $\mathrm{OH}$ 44023-4398

Contact: Tom Litchney

(800) 289-7326

FAX: (216) 543-5944

Category: RA7

Techno Sommer Automatic

2101 Jericho Turnpike

New Hyde Park, NY 11042-5416

(516) 328-3970

FAX: (516) 326-8827

Category: RA7

Viking Systems International 2070 William Pitt Way

Pittsburgh, PA 15238

Contact: Jack Saluja

(412) 826-3355

FAX: (412) 826-3353

Category: RA5
Visual Inspection Technologies

27-2 Ironia Road

Flanders, NJ 07836-9124

Contact: Bruce Pellegrino

(800) 848-5665

FAX: (201) 927-3207

Category: RA5

Welding Services, Inc.

2225 Skyland Court

Norcross, GA 30071

Contact: Mike Welch

(800) 868-9353

FAX: (404) 449-4684

Category: RA7

Western Space and Marine

111 Santa Barbara Street

Santa Barbara, CA 93101

Contact: Jeff Millard

(805) 963-3831

FAX: (805) 963-3832

Category: RA1, RA5

Westinghouse

Waltz Mill Site

Madison, PA 15663

Contact: Tom Arzenti

(412) 722-5845

Category: RA7

R. W. Wiesener, Inc.

705 Brunson Drive

Albermarle, NC 28001

(704) $982-9242$

FAX: (704) 982-9242

Category: RA7 
APPENDIX B:

CRANE AND GANTRY SUPPLIERS 
APPENDIX B:

GRANE AND GANTRY SUPPLIERS

TABLE B.1 Categories Used to Describe Cranes/Gantries

\begin{tabular}{|l|l|}
\hline Category & \multicolumn{1}{|c|}{ Description } \\
\hline CG1 & Design and build: full range \\
\hline CG2 & Design and build: up to 50 tons \\
\hline CG3 & Hydraulic jack gantries \\
\hline CG4 & Standard product line: special use \\
\hline CG5 & Booms and lifts commercially available/customized \\
\hline CG6 & None of the above \\
\hline
\end{tabular}

Abell-Howe Co.

7747 Van Buren Street

Forest Park, IL 60130

Contact: Tony Pabich

(708) 366-4800

FAX: (708) 366-3401

Category: CG2

AEA Technology

241 Curry Hollow Road

Pittsburgh, PA 15236-2928

Contact: Tim Boorman

(412) 655-1200

FAX: (412) 655-2928

Category: CG2

American Crane \& Equipment Corp.

(ACECO)

531 Old Swede Road

Douglassville, PA 19518

Contact: David Schaeffer

(610) 385-6061

FAX: (610) 385-3191

Category: CG1
Applied Radiological Control

500 Chastain Center Road

Kennesaw, GA 30144-5999

Contact: Ted Martucci

(800) 241-6575

FAX: (404) 428-3090

Category: CG6

The Bartholomew Co.

91 New York Ave.

Westbury, NY 11590

Contact: Harry G. Gotte III

(516) 333-0100

FAX: (516) 333-0301

Category: CG2

B.E. Wallace Products Corp.

71 Bacton Hill Road

Frazer, PA 19355

Contact: Sherry de Carville

(610) 647-1400

FAX: (610) 644-9043

Category: CG4 
Brokk

1144 Village Way

Monroe, WA 98272

Contact: Mike Martin

(800) 621-7856

FAX: (206) 487-2963

Category: CG5

\section{Cattron}

58 W. Shewago

Pittsburgh, PA 16150

Contact: Brian Ellison

(412) 962-3571

FAX: (412) $962-4310$

Category: CG6

Ederer Inc.

2925 First Ave. South

Seattle, WA 98134

Contact: Brian Kennon

(206) 622-4421

FAX: (206) 623-8583

Category: CG1

Flow International

23500 64th Ave.

South Kent, WA 98032

Contact: Mark Fleck

(612) 440-6016

FAX: (206) 813-3285

Category: CG4

4 Point Lift Systems

205 41st Street

P.O. Box 906

Moline, IL 61265

Contact: Bruce Foster

(800) 728-4911

FAX: (309) 764-9848

Category: CG3, CG5
Grove Worldwide

1565 Buchanan Trail East

P.O. Box 21

Shady Grove, PA 17256-0021

Contact: Traci Hahn

(717) 597-8121

FAX: (717) 597-4062

Category: CG5

Harnischweger

P.O. Box 554

Milwaukee, WI 53201

Contact: Ray Greene

(414) 671-4400

FAX: (414) 671-7469

Category: CG1

Hydro-Abrasive Machining Inc.

8831 Miner St.

Los Angeles, CA 9002

Contact: Mike Woolman

(213) $587-1342$

FAX: (213) 587-1818

Category: CG6

IMPSA International, Inc.

1910 Cochran Road

Pittsburgh, PA 15220

Contact: Gerald Katz

(412) 344-7003

FAX: (412) 344-7009

Category: CG1

J\&R Engineering Company, Inc. W224 S8535 Industrial Drive

P.O. Box 218

Big Bend, WI 53103

Contact: Roger Johnston

(414) 662-2297

FAX: (414) 662-4039

Category: CG3, CG5 
Jervis B. Webb Co.

34375 West Twelve Mile

Farmington Hills, MI 48331

Contact: Paul Hopersberger

(810) $553-1220$

FAX: (810) 553-1228

Category: CG6

Lift Tech Cranes

4141 W. Broadway

Muskegon, MI 49444

Contact: Brian Reed (616) 733-0821

Bill Cozzens (708) 968-0802

FAX: (616) 739-7249

Category: CG6

Mi-Jack

3111 W. 167th Street

Hazelcrest, IL 60429

Contact: Don Fox or Dan Heraty

(708) $596-5200$

FAX: (708) 225-2312

Category: CG4, CG5

NES, Inc.

44 Shelter Rock Road

Danbury, CT 06810-7095

Contact: Jim Hedtke

(203) 796-5273

FAX: (203) 796-3168

Category: CG6

PaR Systems, Inc.

899 Highway 96 West

Shoreview, MN 55126

Contact: Albert J. Sturm, Jr.

(612) 484-7261

FAX: (612) 483-2689

Category: CG2
RedZone Robotics, Inc.

2425 Liberty Avenue

Pittsburgh, PA 15222-4639

Contact: Leona Bares

(412) 765-3064

FAX: (412) 765-3069

Category: CG5

Resource Services International 6001 Breakwater Avenue

Cleveland, $\mathrm{OH} 44102$

Contact: Harald C. Pedersen

(216) $651-2550$

FAX: (216) 651-6811

Category: CG5

Rigging International

P.O. Box 4013

Alameda, CA 94501-1029

Contact: Jeanette Mungai

(510) $865-2400$

FAX: (510) $865-9450$

Category: CG6

Stock Equipment Co.

16490 Chillicothe Road

Chagrin Falls, $\mathrm{OH}$ 44023-4398

Contact: Tom Litchney

(800) 289-7326

FAX: (216) 543-6678

Category: CG2

2 DM Associates

7322 Southwest Expressway

Suite 670

Houston, TX 77074

Contact: David Duerr

(713) 270-5044

FAX: (713) 270-6139

Category: CG6 
United States Crane Inc.

P.O. Box 593290

Orlando, FL 32859-3290

Contact: Dean German

(407) $859-6000$

FAX: (407) 857-9146

Category: CG6

Western Space and Marine

111 Santa Barbara Street

Santa Barbara, CA 93101

Contact: Jess Millard

(805) 963-3831

FAX: (805) 963-3832

Category: CG-5

Whiting Corporation

15700 Lathrop Ave.

Harvey, IL 60426-5198

Contact: Greg Ciecierski

(312) 468-9400

FAX: (708) 210-5030

Category: CG1
Williams Crane \& Rigging, Inc.

P.O. Box 1116

Richmond, VA 23208

Contact: Carl Schmidt

(804) 233-9221

FAX: (804) 232-4121

Category: CG6

Zenar

7301 S. 6th Street

Oak Creek, WI 53154

Contact: Bill Kenton

(414) $764-1800$

FAX: (414) 764-1267

Category: CG1

Zimmerman Handling Systems

29555 Stephenson Hwy.

Madison Heights, MI 48071-2387

Contact: Steve Calvert

(708) 231-9301

FAX: (810) 398-1374

Category: CG4 
APPENDIX C:

GLOSSARY 


\section{APPENDIX C: GLOSSARY}

Bridge Crane

CCTV

Computer Control

Commercial

Degrees of Freedom (DOF)

End-Effector

Force Feedback

Gantry Crane

Hoist

Joystick

Manipulator

Master/Slave
A crane with a movable bridge that travels on an overhead fixed runway. The bridge supports a fixed or movable trolley that carries the hoisting mechanism.

Closed-circuit television.

Use of a computer, with or without human intervention, to control movements and operations of equipment. The computer can be preprogrammed or it can "learn" as an operation is under way.

An item offered as a standard item for sale (usually a catalog item).

A direction or dimension of motion in a mechanism, such as wrist rotation or elbow pivot.

An accessory device or tool specifically designed for attachment to a robot wrist or tool mounting plate to enable the robot to perform its intended task.

The human operator of the master arm actually feels a portion of the load as sensed by each joint of a robotic arm.

A crane similar to a bridge crane, except the bridge for supporting the trolley is supported on two or more legs running on a runway.

A machinery unit that is used for lifting or lowering a freely suspended load.

A device consisting of a hand-held stick that pivots about one end and transmits to a computer its angle in two dimensions..

Servo-controlled, open-link, series connected, kinematic chains that perform sophisticated 3-D motion. "Manipulators are sometimes referred to as "robotic arms."

Term generally applied only to the common mechanical and electronic manipulators that have long been used in hot cells. The controls are the master and the actuators are the slave. 
Payload

Programmable

Remotely Operated

Robotic Device

Teach/Learn (Teach/Repeat) The generation and storage of a series of positional data points

Telemanipulation

Teleoperation

Telerobotics

Virtual Reality affected by moving the manipulator through a path of intended motions.

The weight of an item, including all rigging and hardware, being lifted by a crane or gantry.

The ability to cause a manipulator or robotic system to perform a repeatable desired function through the use of a series of predetermined and prerecorded commands.

The ability to operate and control a mechanical device from a remote location by either human or computer control.

A multi-purpose mechanical manipulator capable of performing a sequence of physical tasks without human intervention.

An approach that uses highly dexterous "extended reach tools" and physical barriers between the human and the hazardous environment. Manipulation is often through mechanical master/slave manipulators, and vision is usually through a window or periscope.

An approach that uses a general purpose, dexterous, humanmachine system that augments a human by projecting his or her manipulatory capabilities across distance and through physical barriers into hostile environments. Typically, manipulation is by movable mechanisms, and vision is through windows, periscopes, or cameras.

An approach that uses teleoperation with selectable automation. Some functions are performed without human intervention.

Computer simulations that use 3D graphics and various devices to allow the user to interact with the simulation.

* Remote Handling Technology: History and State-of-the-Art, 1987, Oak Ridge National Laboratory, Oak Ridge, TN. 
NOTE: This Distribution List is in addition to all of the organizations listed in the two Vendor Contacts Lists in the report.

\section{DISTRIBUTION LIST}

\section{Internal}

Aldana, $\mathrm{J}$.

Ahluwalia, R.

Bailey, J.

Bhattacharyya, S.K.

Black, D.

Boing, L.

Brunner, $F$.

Coffey, $\mathrm{M}$.

Collins, E.

Ditch, R.

Fellhauer, C.

Fink, C.

Grandy, C.

Helt, J.

Henley, D.

Klotz, C.E.

Knox, A.

Leong, $\mathrm{K}$.

Lineberry, $M$.

McFarlane, $\mathrm{H}$.

Mezaraups, J.

Micklich, B.

Mosho, G.

Novick, V.

Pancake, D.

Pfeiffer, $P$.

Picologlou, B.

Purohit, A.

Reed, C.

Rose, $\mathrm{R}$.

Sodaro, $\mathrm{M}$.
Seifert, L.

Seiford, R.

Smith, R.S.

Surles, T.

Yule, $T$.

ANL Libraries (Bldg. 203)

D\&D Library (Bldg. 207)

\section{DOE-CH/ARG}

Baker, D.

Crawford, J.

Gabel, A.

Haugen, J.

Taboas, A.

Webster, S.

Wunderlich, R.C.

Zeigler, B.

DOE-HQ

EM-40

Baubitz, J.

Robeson, S.

EM-50

Frank, C.

Hyde, J.

Lien, S.

Lilly, J.

Yarbrough, L. 
External

DOE-METC, 3610 Collins Ferry Rd., Morgantown, WV 26507

Bechtel, S.

Beddick, B.

Bossart, $\mathrm{S}$.

Hart, P

Advanced Consulting Group, Inc. (ACG)

Thomas J. Litka, Executive Towers, Suite 603, 5901 North Cicero Avenue, Chicago, IL 60646

Energy Technology Engineering Center (Rockwell International Corp.)

Rod Meyer, ETEC, P.O. Box 7930, Canoga Park, CA 9.1309

G. Subbaramen, ETEC, P.O. Box 7930, Canoga Park, CA 91309

Idaho National Engineering Laboratory (INEL), operated by Lockheed Martin Idaho Technologies Co. (LMIT)

Kevin Croft, P.O. Box 1625, Idaho Falls, ID 83415

Dick Meservey, P.O. Box 1625, Idaho Falls, ID 83415

Bruce M. Wilding, P.O. Box 1626, Mail Stop 2220, Idaho Falls, ID 83415

Walter D. (David) Willis, P.O. Box 1626, Mail Stop 2220, Idaho Falls, ID 83415

Quest Integrated, Inc. (QI2)

Edward Bohn, 21414 68th Avenue South, Kent, WA 98032

Bruce Goldwater, 21414 68th Avenue South, Kent, WA 98032

Oak Ridge National Laboratory (ORNL) operated by Lockeed Martin Energy Systems, Inc.

Tom Burwinle, P.O. Box 2008, Building 3001, Oak Ridge, TN 37831-6029

Dennis C. Haley, P.O. Box 2008, Building 7601, Oak Ridge, TN 37831-6304

William R. Hamel, P.O. Box 2008, Building 7601, Oak Ridge, TN 37831-6304

Charles Hensley, P.O. Box 2003, Building K-1580, Oak Ridge, TN 37831

John Kennerly, P.O. Box 2003, Oak Ridge, TN 37831-7298

Mark W. Noakes, P.O. Box 2008, Building 7601, Oak Ridge, TN 37831-6304

Park T. Owen, RAPIC, 138 Mitchell Rd., Oak Ridge, TN 37830-7918

\section{Redzone Robotics, Inc.}

Leona (Lee) C. Bares, 2425 Liberty Avenue, Pittsburgh, PA 15222-4639

Lou Conley, 2425 Liberty Avenue, Pittsburgh, PA 15222-4639

Bruce Thompson, 2425 Liberty Avenue, Pittsburgh, PA 15222-4639 
Sandia National Laboratories (SNL)

Brady R. Davies, P.O. Box 5800, Albuquerque, NM 87185-1177

Dr. James P. Hickerson, Jr., P.O. Box 5800, Albuquerque, NM 87185-1177

Daniel S. Horschel, P.O. Box 5800, Albuquerque, NM 87185-1177

Waste Policy Institute (WPI)

Ken Kasper, Waste Policy Institute, 1224 Pinview Drive, Morgantown, WV 26505

Robert Vagnetti, 1224 Pinview Drive, Morgantown, WV 26505

Westinghouse Savannah River Company

Bill Austin, 1991 South Centennial Ave., Building \#1, Aikin, SC 29803

Rand (Randy) P. Singer, P.O. Box 616, Building 773A, Aiken, SC 29808

Clyde R. Ward, P.O. Box 616, Building 773A, Aiken, SC 29808

Eric Kriikku, P.O. Box 616, Building 773A, Aiken, SC 29808

Brad Jenkins, Building 773-A, Room D-1132, Aiken, SC 29808

\section{University of Texas}

S. Tonsunoglu, University of Texas, Austin, TX

\section{Westinghouse Hanford Company}

Eric Shen, P.O. Box 1970 R3-86, Richland, WA 99352 
